\title{
Orthogonal product bases of four qubits
}

\author{
Lin Chen ${ }^{1,2, *}$ and Dragomir Ž Đoković ${ }^{3, \dagger}$ \\ ${ }^{1}$ School of Mathematics and Systems Science, \\ Beihang University, Beijing 100191, China \\ ${ }^{2}$ International Research Institute for Multidisciplinary Science, \\ Beihang University, Beijing 100191, China \\ ${ }^{3}$ Department of Pure Mathematics and Institute for Quantum Computing, \\ University of Waterloo, Waterloo, Ontario, N2L 3G1, Canada
}

(Dated: September 7, 2018)

An orthogonal product basis $(\mathrm{OPB})$ of a finite-dimensional Hilbert space $\mathcal{H}=\mathcal{H}_{1} \otimes$ $\mathcal{H}_{2} \otimes \cdots \otimes \mathcal{H}_{n}$ is an orthonormal basis of $\mathcal{H}$ consisting of product vectors $\left|x_{1}\right\rangle \otimes\left|x_{2}\right\rangle \otimes$ $\cdots \otimes\left|x_{n}\right\rangle$. We show that the problem of constructing the OPBs of an $n$-qubit system can be reduced to a purely combinatorial problem. We solve this combinatorial problem in the case of four qubits and obtain 33 multiparameter families of OPBs. Each OPB of four qubits is equivalent, under local unitary operations and qubit permutations, to an OPB belonging to at least one of these families.

* linchen@buaa.edu.cn (corresponding author)

† djokovic@uwaterloo.ca 


\section{CONTENTS}

I. Introduction

II. Orthogonal product bases

III. OPBs of multiqubit systems

V. Classification of four-qubit orthogonal product bases

A. Preliminaries

B. Main result

VI. Applications

41

A. Construction of OPBs in higher dimensions

41

B. Weak equivalence and controlled unitary operations

42

VII. Discussion

43

Acknowledgements

44

Appendix

44

References

49

\section{INTRODUCTION}

The local operations and classical communication (LOCC) are the fundamental measurements for many quantum-information protocols and problems [1]. The quantum teleportation is carried out using LOCC and quantum entanglement [2], the well-known distillability problem [3, 4] and distinguishing of quantum states are investigated under LOCC [5]. The LOCC-indistinguishable product states imply the quantum nonlocality without entanglement [6]. It is known that the unextendible product bases (UPBs) [6] and irreducible orthogonal product bases (OPBs) [9, Theorem 3] are LOCC-indistinguishable. (For the definition of reducible and irreducible OPBs see section [II) The irreducible three-qubit and 
two-qutrit OPBs have been classified in [9].

The difficulty of constructing and classifying multiqubit OPBs increases rapidly with the number of qubits. We show in this paper how the construction problem can be reduced to a purely combinatorial problem. In the case of four qubits, by solving the latter problem, we provide a method for the construction of all OPBs. The combinatorial problem deals with the special kind of $2^{n} \times n$ matrices $M \in \mathcal{O}(n)$ where $n$ is the number of qubits (see Sec. III for the precise definition of $\mathcal{O}(n)$.) The entries of $M$ are formal variables $a, b, \ldots$, which represent unit vectors of various one-qubit Hilbert spaces $\mathcal{H}_{j}, j=1, \ldots, n$. Each of these vector variables $a$ has as a companion another vector variable $a^{\perp}$. To a vector variable $a$ in column $j$ we may assign as values arbitrary unit vectors $|\alpha\rangle$ in $\mathcal{H}_{j}$. Then to $a^{\perp}$ we have to assign the value $|\alpha\rangle^{\perp} \in \mathcal{H}_{j}$. After assigning the unit vector values to each vector variable of $M$, we obtain an OPB by simply taking the tensor product of the unit vectors in each row. Thus each $M$ determines an infinite family of OPBs which we denote by $\mathcal{F}_{M}$. A family $\mathcal{F}_{M}$ is called maximal if it is not a proper subset of another family $\mathcal{F}_{N}$. (This is equivalent to our formal definition (7)

We say that two matrices are equivalent if the corresponding families of OPBs are equivalent by local unitary operations and qubit permutations. (Although our formal definition of equivalence of matrices $M$ is different, see Definition [6, one can show that it is equivalent to this one.) The construction of OPBs reduces to the classification of maximal matrices $M$ up to equivalence. For convenience of the latter classification, we introduce weak equivalence in the set of maximal matrices and refer to weak equivalence classes as switching classes. This is the key notion for simplifying the enumeration of the equivalence classes in $\mathcal{O}(n)$. Each switching class is a union of ordinary equivalence classes.

There are infinitely many equivalence classes of OPBs of $n$ qubits (up to local unitary transformations and qubit permutations). On the other hand there are only finitely many equivalence classes of matrices in $\mathcal{O}(n)$. Given an $\mathrm{OPB}$, say $\mathcal{A}$, after ordering its product vectors we can associate to it in a natural way a matrix $M \in \mathcal{O}(n)$ such that $\mathcal{A} \in \mathcal{F}_{M}$ (see the end of section (III). Thus the set of all OPBs is the union of finitely many families $\mathcal{F}_{M}$. Note that the matrix $M$ is not uniquely determined by $\mathcal{A}$ as it depends on the choice of ordering of $\mathcal{A}$ and naming of the vector variables when constructing $M$. However, all $M$ s that we get in this way are equivalent to each other. Further, if $U$ is any local unitary operator then we have $U \mathcal{A} \in \mathcal{F}_{M}$. Hence, the equivalence class of $M$ depends only on the equivalence class 
of $\mathcal{A}$. In other words, we obtain a well defined map from the set of equivalence classes of OPBs of $n$ qubits to the set of equivalence classes of $\mathcal{O}(n)$. This mapping is onto but, of course, not one-to-one.

To construct all OPBs, instead of arbitrary matrices $N \in \mathcal{O}(n)$ one can use just the maximal ones. More precisely, let $M_{1}, M_{2}, \ldots, M_{k}$ be the list of representatives of the equivalence classes of maximal matrices in $\mathcal{O}(n)$. Then any $\mathrm{OPB} \mathcal{A}$ is equivalent to one in some $\mathcal{F}_{M_{i}}$. The main result of this paper (Theorem 19) provides such a list in the case of four qubits. However, we did not compute the list of all equivalence classes. (In principle such computation can be performed, say by a computer, but writing an efficient program is a challenge.) In the case of three qubits we computed the list of all equivalence classes (see Lemma 14 and its corollary).

In addition to constructing the OPBs we can also obtain a very coarse classification of them. Let $M \in \mathcal{O}(n)$ be the matrix associated to $\mathcal{A}$. If $\mathcal{A} \in \mathcal{F}_{N}$ for some $N \in \mathcal{O}(n)$ then $\mathcal{F}_{M} \subseteq \mathcal{F}_{N}$. Thus, among all families $\mathcal{F}_{N}$ containing $\mathcal{A}$ there is the smallest one, namely $\mathcal{F}_{M}$. In general, the condition $\mathcal{A} \in \mathcal{F}_{N}$ does not determine $N$ uniquely. A slight modification can correct this default. For that purpose we introduce the strict families $\mathcal{F}_{M}^{\#}$. In order to define strict families we introduce a (non-transitive) binary relation, denoted by " $<$ ", on $\mathcal{O}(n)$ (see section 【II). If $N<M$ then $\mathcal{F}_{N} \subset \mathcal{F}_{M}$. (If there is no $N$ such that $M<N$ then $M$ is maximal.) By definition, $\mathcal{F}_{M}^{\#}$ consists of all OPBs in $\mathcal{F}_{M}$ which do not belong to any $\mathcal{F}_{N}$ with $N<M$. We still have that $\mathcal{A} \in \mathcal{F}_{M}^{\#}$. Moreover, $\mathcal{F}_{M}^{\#}$ is the unique strict family containing $\mathcal{A}$.

Let $\mathcal{R}$ be a set of representatives of the equivalence classes of $\mathcal{O}(n)$, a finite set. Our proposed classification is a partition of the set of all OPBs into finite number of classes, one for each $M \in \mathcal{R}$. The class corresponding to $M \in \mathcal{R}$ consists of all OPBs which are equivalent to an OPB belonging to the strict family $\mathcal{F}_{M}^{\#}$.

In the case of three qubits, there exists exactly one reducible and one irreducible switching class of maximal matrices (see Lemma 14). In the case of four qubits we prove in Theorem 19 that there are in total 17 switching classes of maximal matrices, and we list their representatives (see (13)-(17)). Among these switching classes, 3 consist of reducible and 12 of irreducible matrices. Two preliminary facts are proven in lemmas [16 and [18. Due to its length, the rest of the proof of Theorem 19 is given in a separate section, $\mathrm{VB}$. When a switching class contains more then one equivalence class, we list their representatives in the 
appendix. In total, there are 33 equivalence classes of maximal matrices.

Throughout this paper we shall use the following notation. Let $\mathcal{H}=\mathcal{H}_{1} \otimes \mathcal{H}_{2} \otimes \cdots \otimes \mathcal{H}_{n}$ be the complex Hilbert space of a finite-dimensional $n$-partite quantum system. We denote by $d_{i}$ the dimension of $\mathcal{H}_{i}$, and so $D:=\prod d_{i}$ is the dimension of $\mathcal{H}$. To avoid trivial cases, we assume that each $d_{i}>1$ and $n>1$. A vector $|x\rangle \in \mathcal{H}$ is normalized if $\|x\|=1$. We denote by $H$ the space of Hermitian operators $\rho$ on $\mathcal{H}$. Note that $H$ is a real vector space of dimension $D^{2}$. The mixed quantum states of this quantum system are represented by their density matrices, i.e., operators $\rho \in H$ which are positive semidefinite $(\rho \geq 0)$ and have unit trace $(\operatorname{Tr} \rho=1)$.

We assume that an orthonormal (o. n.) basis is fixed in each $\mathcal{H}_{i}$ and we use the standard notation $|0\rangle, \ldots,\left|d_{i}-1\right\rangle$ for the corresponding basis vectors. A product vector is a nonzero vector of the form $|x\rangle=\left|x_{1}\right\rangle \otimes \cdots \otimes\left|x_{n}\right\rangle$ where $\left|x_{i}\right\rangle \in \mathcal{H}_{i}$. We shall write this product vector also as $\left|x_{1}, \ldots, x_{n}\right\rangle$. When $\left|x_{1}, \ldots, x_{n}\right\rangle$ is a unit vector, we shall also assume that each $\left|x_{i}\right\rangle$ is a unit vector. A pure product state is a state $\rho$ of the form $\rho=|x\rangle\langle x|$ where $|x\rangle$ is a product vector. The product vectors $\left|i_{1}, i_{2}, \ldots, i_{n}\right\rangle, 0 \leq i_{k}<d_{k}$, form an o. n. basis of $\mathcal{H}$. We refer to this basis as the standard basis.

\section{ORTHOGONAL PRODUCT BASES}

We say that an orthonormal basis of $\mathcal{H}$ consisting of product vectors,

$$
\mathcal{A}:=\left\{\left|a_{s}\right\rangle=\left|a_{s, 1}, \ldots, a_{s, n}\right\rangle: s=1, \ldots, D\right\},
$$

is an orthogonal product basis (OPB).

The OPBs can be divided into reducible and irreducible ones. We say that the OPB $\mathcal{A}$ is reducible if for some $i \in\{1,2, \ldots, n\}$ there exists a partition of $\{1,2, \ldots, D\}$ into two nonempty parts $J$ and $K$ such that $\left|a_{j, i}\right\rangle \perp\left|a_{k, i}\right\rangle$ for all $j \in J$ and $k \in K$. An OPB is irreducible if it is not reducible.

Assume that $\mathcal{A}$ is reducible and let $i, J, K$ be as above. Denote by $\mathcal{H}_{i, J}$ the subspace of $\mathcal{H}_{i}$ spanned by the vectors $\left|a_{j, i}\right\rangle$ with $j \in J$, and define $\mathcal{H}_{i, K}$ similarly. Denote by $\mathcal{H}^{\prime}$ the subsystem $\mathcal{H}_{1} \otimes \cdots \otimes \mathcal{H}_{i, J} \otimes \cdots \otimes \mathcal{H}_{n}$ of $\mathcal{H}$, and define $\mathcal{H}^{\prime \prime}$ similarly (by replacing $J$ with $K$ ). Clearly, $\mathcal{A}$ is the union of an OPB of $\mathcal{H}^{\prime}$ and an OPB of $\mathcal{H}^{\prime \prime}$. This shows that the description of the OPBs of the system $\mathcal{H}$ reduces to the case of irreducible OPBs (modulo the systems 
of lower dimension). The irreducible OPBs in the case of two qutrits and three qubits have been classified in [9].

For a nonzero vector $|x\rangle \in \mathcal{H}_{i}$, we denote by $[x]$ the one-dimensional subspace spanned by this vector. If $\left\{\left|x_{j}\right\rangle: j=1, \ldots, d_{i}\right\}$ is a basis of $\mathcal{H}_{i}$, then we say that

$$
F:=\left\{\left[x_{j}\right]: j=1,2, \ldots, d_{i}\right\}
$$

is a frame of $\mathcal{H}_{i}$. If moreover the $\left[x_{j}\right]$ are pairwise orthogonal, we say that $F$ is an orthogonal frame.

For the OPB $\mathcal{A}$ given by (1), we set

$$
\mathcal{A}_{i}=\left\{\left[a_{s, i}\right]: s=1, \ldots, D\right\}, \quad i=1, \ldots, n .
$$

For any 1-dimensional subspace $V \subseteq \mathcal{H}_{i}$ we define its multiplicity $\mu(V)$ to be the number of indices $s \in\{1,2, \ldots, D\}$ such that $\left[a_{s, i}\right]=V$. In particular, $\mu(V)=0$ if $\left[a_{s, i}\right] \neq V$ for all indices $s$.

Proposition 1 Suppose that $d_{1}=2$ and set $\mathcal{H}^{\prime}=\mathcal{H}_{2} \otimes \cdots \otimes \mathcal{H}_{n}$. Let $\mathcal{A}$ be the OPB given by (11) and define the $\mathcal{A}_{i}$ by (2). For each $s \in\{1,2, \ldots, D\}$ set $\left|a_{s}^{\prime}\right\rangle:=\left|a_{s, 2}, a_{s, 3}, \ldots, a_{s, n}\right\rangle$. Let $\mathcal{E}=\left\{V_{1}, V_{2}, \ldots, V_{m}\right\} \quad\left(V_{j}\right.$ distinct) be any maximal subset of $\mathcal{A}_{1}$ not containing any orthogonal frame. Then the following hold.

(i) $\mathcal{A}_{1}$ is the disjoint union of orthogonal frames $F_{j}=\left\{V_{j}, V_{j}^{\perp}\right\}, j=1,2, \ldots, m, \mu\left(V_{j}\right)=$ $\mu\left(V_{j}^{\perp}\right)$ for each $j$, and the vectors $\left|a_{s}^{\prime}\right\rangle$ for which $\left[a_{s, 1}\right] \in \mathcal{E}$ form an OPB of $\mathcal{H}^{\prime}$.

(ii) If $P_{j}=\left\{s:\left[a_{s, 1}\right]=V_{j}\right\}$ and $Q_{j}=\left\{s:\left[a_{s, 1}\right]=V_{j}^{\perp}\right\}$ then $\sum_{s \in P_{j}}\left[a_{s}^{\prime}\right]=\sum_{s \in Q_{j}}\left[a_{s}^{\prime}\right]$.

(iii) If all $d_{i}=2$ then $\sum_{i=1}^{n} \mu\left(\left[a_{s, i}\right]\right) \geq D-1$ for each $s \in\{1,2, \ldots, D\}$.

Proof. (i) Let us first assume that $\mathcal{E}$ is chosen so that $\mu\left(V_{j}\right) \geq \mu\left(V_{j}^{\perp}\right)$ for each $j$. The maximality property implies that for each $V \in \mathcal{A}_{1} \backslash \mathcal{E}$ we have $V^{\perp} \in \mathcal{E}$. Let $\mu^{\prime}=\sum_{V \in \mathcal{E}} \mu(V)$ and $\mu^{\prime \prime}=\sum_{V \in \mathcal{E}} \mu\left(V^{\perp}\right)$. Note that $\mu^{\prime}+\mu^{\prime \prime}=\sum_{V \in \mathcal{A}_{1}} \mu(V)=D$ and $\mu^{\prime}-\mu^{\prime \prime}=\sum_{V \in \mathcal{E}}(\mu(V)-$ $\mu\left(V^{\perp}\right) \geq 0$. If $\left[a_{s, 1}\right],\left[a_{t, 1}\right] \in \mathcal{E}, s \neq t$, then $\left\langle a_{s, 1} \mid a_{t, 1}\right\rangle \neq 0$ and so $\left|a_{s}^{\prime}\right\rangle \perp\left|a_{t}^{\prime}\right\rangle$. It follows that $\sum\left[a_{s}^{\prime}\right]$, taken over all indices $s$ for which $\left[a_{s, 1}\right] \in \mathcal{E}$, has dimension $\mu^{\prime}$. Consequently, $\mu^{\prime} \leq \operatorname{Dim} \mathcal{H}^{\prime}=D / 2$. Hence, $2 \mu^{\prime} \leq D=\mu^{\prime}+\mu^{\prime \prime}$, i.e., $\mu^{\prime} \leq \mu^{\prime \prime}$. As also $\mu^{\prime \prime} \leq \mu^{\prime}$, we have $\mu^{\prime}=\mu^{\prime \prime}$. It follows that for each $V \in \mathcal{E}$ we have $\mu(V)=\mu\left(V^{\perp}\right)$, and so $V^{\perp} \in \mathcal{A}_{1}$. Consequently, all possible maximal subsets $\mathcal{E} \subseteq \mathcal{A}_{1}$ not containing any orthonormal frame satisfy the additional assumption made at the beginning of the proof. We can now drop that assumption. 
The first two assertions of (i) have been proved. The third follows from the fact shown above that $\left|a_{s}^{\prime}\right\rangle \perp\left|a_{t}^{\prime}\right\rangle$ provided that $\left[a_{s, 1}\right],\left[a_{t, 1}\right] \in \mathcal{E}$ and $s \neq t$.

(ii) From (i) we know that $\mathcal{H}^{\prime}$ is an orthogonal direct sum of the subspaces $X_{j}:=$ $\sum_{s \in P_{j}}\left[a_{s}^{\prime}\right], j=1,2, \ldots, m$, and also orthogonal direct sum of the subspaces $Y_{j}:=\sum_{s \in Q_{j}}\left[a_{s}^{\prime}\right]$, $j=1,2, \ldots, m$. Since $\mu\left(V_{k}\right)=\mu\left(V_{k}^{\perp}\right)$ we can interchange the roles of $V_{k}$ and $V_{k}^{\perp}$ for a single index $k$. Hence $Y_{k}$ is also the orthogonal complement of $\sum_{j \neq k} X_{j}$ and so $Y_{k}=X_{k}$.

(iii) For each $t \neq s$ there is at least one $i$ such that $\left\langle a_{s, i} \mid a_{t, i}\right\rangle=0$. Let $J_{i}$ be the set of indices $t$ such that $\left\langle a_{s, i} \mid a_{t, i}\right\rangle=0$. As all $d_{i}=2,\left\langle a_{s, i} \mid a_{t, i}\right\rangle=0$ is equivalent to $\left[a_{t, i}\right]=\left[a_{s, i}\right]^{\perp}$. Since $\mu\left(\left[a_{s, i}\right]\right)=\mu\left(\left[a_{s, i}\right]^{\perp}\right)$, we have $\left|J_{i}\right|=\mu\left(\left[a_{s, i}\right]\right)$. As $\mathcal{A}$ is an OPB, we have $\cup_{i=1}^{n} J_{i}=\{1,2, \ldots, D\} \backslash\{s\}$. Hence $\sum_{i=1}^{n} \mu\left(\left[a_{s, i}\right]\right)=\sum_{i=1}^{n}\left|J_{i}\right| \geq D-1$.

It follows from this proposition that in the bipartite systems with $d_{1}=2$ we can construct all OPBs by the following method. We choose an orthogonal decomposition $\mathcal{H}_{2}=X_{1} \oplus \cdots \oplus$ $X_{m}$ and for each $j \in\{1,2, \ldots, m\}$ we choose a unit vector $v_{j} \in \mathcal{H}_{1}$ and two arbitrary o.n. bases $\left\{x_{j, 1}, x_{j, 2}, \ldots, x_{j, k_{j}}\right\}$ and $\left\{y_{j, 1}, y_{j, 2}, \ldots, y_{j, k_{j}}\right\}$ of $X_{j}$. Then the $D$ product vectors

$$
\begin{aligned}
& \left|v_{j}, x_{j, 1}\right\rangle, \ldots,\left|v_{j}, x_{j, k_{j}}\right\rangle, \\
& \left|v_{j}^{\perp}, y_{j, 1}\right\rangle, \ldots,\left|v_{j}^{\perp}, y_{j, k_{j}}\right\rangle, \\
& j=1, \ldots, m,
\end{aligned}
$$

form an OPB of $\mathcal{H}$.

Corollary 2 Any OPB of the bipartite system $2 \otimes d_{2}$ is reducible.

Proof. The assertion is obvious if $m=1$. If $m>1$ it follows from the observation that the first $2 k_{1}$ product vectors in the above list (those with $j=1$ ) are orthogonal to all the remaining product vectors in the list.

This result also follows from [9, Theorem 3], which says that any irreducible OPB is LOCC-indistinguishable, and the known fact that any $2 \otimes d_{2}$ OPB is LOCC-distinguishable (see the end of [10]). It does not extend to other bipartite systems. For instance, in the case $d_{1}=d_{2}=3$ there exist irreducible OPBs $[9$, Fig 1$]$.

In Proposition 1 (i), $\mathcal{A}_{2}$ is not necessarily a disjoint union of orthogonal frames. For instance, let $d_{2}=3$ and consider the OPBs of the form $|0,0\rangle,|0,1\rangle,|0,2\rangle,|1,0\rangle,|1, x\rangle,|1, y\rangle$ with $[x] \neq[1],[2]$. 


\section{OPBS OF MULTIQUBIT SYSTEMS}

In this section we reduce the classification problem of OPBs in multiqubit systems to a purely combinatorial problem. Thus we set $d_{1}=d_{2}=\cdots=d_{n}=2$ and so $D=2^{n}$.

Given a unit product vector $\left|x_{1}, x_{2}, \ldots, x_{n}\right\rangle$, we shall always assume (as we may) that the vectors $\left|x_{j}\right\rangle$ are unit vectors. For convenience, in this section we shall not distinguish two unit vectors in $\mathcal{H}_{j}$ which differ only by a phase factor, i.e., we consider these vectors as points of the complex projective line $\mathbf{P}\left(\mathcal{H}_{j}\right)$ associated to $\mathcal{H}_{j}$. If $|x\rangle \in \mathcal{H}_{j}$ is a unit vector, then by using this convention, we can say that there exists a unique unit vector $|x\rangle^{\perp} \in \mathcal{H}_{j}$ which is orthogonal to $|x\rangle$. We refer to $|x\rangle^{\perp}$ as the perpendicular of $|x\rangle$.

Let $\mathrm{U}\left(\mathcal{H}_{i}\right)$ be the unitary group of the 2-dimensional Hilbert space $\mathcal{H}_{i}$. For $U_{i} \in \mathrm{U}\left(\mathcal{H}_{i}\right)$, $i=1,2, \ldots, n$, let $U=\left(U_{1}, U_{2}, \ldots, U_{n}\right)$ be the corresponding element of the direct product of the groups $\mathrm{U}\left(\mathcal{H}_{i}\right)$. Then $U$ acts on $\mathcal{H}$ as the local unitary operator $U_{1} \otimes U_{2} \otimes \cdots \otimes U_{n}$. Thus if $|x\rangle=\left|x_{1}, x_{2}, \ldots, x_{n}\right\rangle$, we have $U|x\rangle=U_{1}\left|x_{1}\right\rangle \otimes U_{2}\left|x_{2}\right\rangle \otimes \cdots \otimes U_{n}\left|x_{n}\right\rangle$. Since we have

fixed o.n. bases in all $\mathcal{H}_{i}$, the symmetric group $\operatorname{Sym}_{n}$ acts on $\mathcal{H}$ by permuting the tensor factors $\mathcal{H}_{i}$. Thus $\pi|x\rangle=\left|x_{\pi^{-1}(1)}, x_{\pi^{-1}(2)}, \ldots, x_{\pi^{-1}(n)}\right\rangle$ for $\pi \in \operatorname{Sym}_{n}$.

Definition 3 We say that two OPBs (or two families of $O P B s$ ) $\mathcal{A}$ and $\mathcal{B}$ are equivalent if there exist a local unitary operator $U$ and a permutation $\pi$ such that $\mathcal{B}=\pi U \mathcal{A}$.

(If $\mathcal{A}$ is a family of OPBs then $\pi U \mathcal{A}$ denotes the family obtained by applying the operator $\pi U$ to each member of the family $\mathcal{A}$.)

We denote by $\mathcal{H}_{\times}$the product of the Hilbert spaces $\mathcal{H}_{i}, \mathcal{H}_{\times}=\mathcal{H}_{1} \times \mathcal{H}_{2} \times \cdots \times \mathcal{H}_{n}$. Further, we denote by $\mathcal{H}_{\times}^{r}$ the product of the $r$ copies of the space $\mathcal{H}_{\times}$.

We can represent an OPB, say $\mathcal{A}=\left\{\left|a_{i, 1}, a_{i, 2}, \ldots, a_{i, n}\right\rangle\right\}$, by the corresponding point of the space $\mathcal{H}_{\times}^{2^{n}}$, i.e., the $2^{n} \times n$ matrix $A$ with rows $\left[\left|a_{i, 1}\right\rangle\left|a_{i, 2}\right\rangle \cdots\left|a_{i, n}\right\rangle\right]$. We are interested in the equivalence classes of OPBs for the equivalence defined above. In particular, this means that we can permute the columns of $A$. Since an OPB is just a set (not an ordered set), we can also permute the rows of $A$. Let us give an example of a local unitary operation. The product vectors $|0,0\rangle,|0,1\rangle,|1,+\rangle,|1,-\rangle$ form an OPB of a 2 -qubit system where $| \pm\rangle=\frac{1}{\sqrt{2}}(|0\rangle \pm|1\rangle)$. After applying a Hadamard gate on the second qubit, one obtains $|0,+\rangle,|0,-\rangle,|1,0\rangle,|1,1\rangle$, which is also an OPB. In this sense, the above two OPBs are locally unitarily equivalent but not obtained by just permuting the rows of $A$. 
We will show that the OPBs occur, up to equivalence, in several infinite families which will be specified by $2^{n} \times n$ matrices $M$ whose entries are unit vectors considered as variables. Let us say that $|v\rangle$ is a vector variable if it runs through all unit vectors in one of the spaces $\mathcal{H}_{j}$. If $|v\rangle$ is a vector variable on $\mathcal{H}_{j}$ then the same is true for its perpendicular. We say that a finite collection of pairwise distinct vector variables on $\mathcal{H}_{j}$ is independent if it does not contain a pair consisting of a vector variable and its perpendicular.

Definition 4 We define $\mathcal{O}=\mathcal{O}(n)$ formally to be the set of $2^{n} \times n$ matrices $M=\left[M_{i, k}\right]$ having the following three properties:

(i) Each entry of the $j$ th column of $M$ is either a (unit) vector variable, say $|a\rangle \in \mathcal{H}_{j}$, or $|a\rangle^{\perp}$. To simplify notation, we shall write just $a$ and $a^{\perp}$, respectively. Note that $\left(a^{\perp}\right)^{\perp}=a$.

(ii) If a vector variable, say $a$, occurs in a column of $M$ then neither a nor $a^{\perp}$ occur in any other column.

(iii) Any two distinct rows of $M$, say rows $i$ and $j$, are orthogonal to each other in the sense that $M_{i, k}=M_{j, k}^{\perp}$ for some $k$.

For a given $M \in \mathcal{O}(n)$, we denote by $\mu(a)$ the number of occurencies of the vector variable $a$ in the matrix $M$. We refer to $\mu(a)$ as the multiplicity of $a$. Thus if a vector variable $a$ does not occur in $M$, then $\mu(a)=0$. We shall prove that $\mu(a)=\mu\left(a^{\perp}\right)$.

Lemma 5 A vector variable a and its perpendicular $a^{\perp}$ occur in $M \in \mathcal{O}(n)$ the same number of times, i.e., we have $\mu(a)=\mu\left(a^{\perp}\right)$.

Proof. Let $\left\{a_{1}, a_{2}, \ldots, a_{m}\right\}$ be a maximal set of independent variables which occur in the first column of $M$. Without any loss of generality, we may assume that this set is chosen so that $\mu\left(a_{i}\right) \geq \mu\left(a_{i}^{\perp}\right)$ for all $i$. Set $\mu^{\prime}:=\sum_{i=1}^{m} \mu\left(a_{i}\right)$ and $\mu^{\prime \prime}:=\sum_{i=1}^{m} \mu\left(a_{i}^{\perp}\right)$ and note that $\mu^{\prime}+\mu^{\prime \prime}=2^{n}$ and $\mu^{\prime} \geq \mu^{\prime \prime}$. Denote by $N$ the submatrix of $M$ obtained by first deleting all rows whose first element is one of the variables $a_{i}^{\perp}$ and then deleting the first column. Thus $N$ has $\mu^{\prime}$ rows and $n-1$ columns. Moreover the rows of $N$ are mutually orthogonal. This implies that $\mu^{\prime} \leq 2^{n-1}$. Hence we must have $\mu^{\prime}=\mu^{\prime \prime}=2^{n-1}$ and $\mu\left(a_{i}\right)=\mu\left(a_{i}^{\perp}\right)$ for each $i$.

To define equivalence of matrices $M \in \mathcal{O}(n)$ we need to rename some vector variables. This may be confusing, so we first describe a simple renaming. Let $a$ be a vector variable which occurs in $M$. Recall that $a$ and $a^{\perp}$ occur only in a single column of $M$, say column 
$j$, and that $\mu(a)=\mu\left(a^{\perp}\right)$. We choose a new vector variable $x$ on $\mathcal{H}_{j}$. Finally, we replace simultaneously each occurrence of $a$ with $x$ and each occurrence of $a^{\perp}$ with $x^{\perp}$. A general renaming is just a composition of finitely many simple renamings. In particular it may be a trivial renaming, which means that we do not make any changes in $M$.

Definition 6 We say that two matrices $M, N \in \mathcal{O}(n)$ are equivalent if $N$ can be obtained from $M$ by permuting rows and columns and renaming of the vector variables. We refer to row permutations, column permutations and renamings as equivalence operations.

We denote by $[A]$ the equivalence class in $\mathcal{O}(n)$ containing the matrix $A \in \mathcal{O}(n)$.

Let us give an example. For instance the matrices $A, X \in \mathcal{O}(2)$ given by

$$
A=\left[\begin{array}{cc}
a & b \\
a & b^{\perp} \\
a^{\perp} & c \\
a^{\perp} & c^{\perp}
\end{array}\right], \quad X=\left[\begin{array}{cc}
x & z \\
y & z^{\perp} \\
y^{\perp} & z^{\perp} \\
x^{\perp} & z
\end{array}\right]
$$

are equivalent, i.e., we have $[A]=[B]$. The reason is that we can transform $A$ to $X$ by the three transformations

$$
\left[\begin{array}{cc}
a & b \\
a & b^{\perp} \\
a^{\perp} & c \\
a^{\perp} & c^{\perp}
\end{array}\right] \rightarrow\left[\begin{array}{cc}
b & a \\
b^{\perp} & a \\
c & a^{\perp} \\
c^{\perp} & a^{\perp}
\end{array}\right] \rightarrow\left[\begin{array}{cc}
b^{\perp} & a \\
c & a^{\perp} \\
c^{\perp} & a^{\perp} \\
b & a
\end{array}\right] \rightarrow\left[\begin{array}{cc}
x & z \\
y & z^{\perp} \\
y^{\perp} & z^{\perp} \\
x^{\perp} & z
\end{array}\right]
$$

The first transformation is a column permutation, the second a row permutation and the third is the renaming of $a, b, c$ to $z, x^{\perp}, y$ respectively.

We say that $M \in \mathcal{O}(n)$ is irreducible if each column of $M$ contains at least two independent vector variables. We say that $M$ is reducible if it is not irreducible.

Let $s_{j}$ be a vector variable on $\mathcal{H}_{j}, j=1,2, \ldots, n$. For $i=1,2, \ldots, 2^{n}$ we write the integer $i-1$ in base 2 as $i-1=\sum_{j=1}^{n} d_{i, j} 2^{j-1}$, where $d_{i, j} \in\{0,1\}$ are the binary digits. We define the standard matrix $S:=\left[s_{i, j}\right] \in \mathcal{O}(n)$ by setting $s_{i, j}=s_{j}$ if $d(i, j)=0$ and $s_{i, j}=s_{j}^{\perp}$ if $d(i, j)=1$. We refer to the equivalence class $[S]$ as the standard class. It is easy to see that all matrices $A \in \mathcal{O}(n)$, which have the property that $\mu(x)=2^{n-1}$ for each entry $x$ of $A$, belong to the standard class. All matrices in this class are obviously reducible.

Let $M \in \mathcal{O}$ and let $a$ and $b$ be independent vector variables which occur in the same column of $M$. Then the matrix obtained from $M$ by setting $b=a$ and $b^{\perp}=a^{\perp}$ everywhere 
in $M$ or by setting $b=a^{\perp}$ and $b^{\perp}=a$ everywhere in $M$ also belongs to $\mathcal{O}$. If $N$ can be obtained from $M$ by this procedure we shall write $N<M$. Note that, according to this definition, $M<N$ and $N<P$ do not imply that $M<P$, i.e., the binary relation " $<$ " is not transitive.

Definition 7 We say that $M \in \mathcal{O}$ is maximal if there is no $N \in \mathcal{O}$ such that $M<N$.

Since each matrix $M \in \mathcal{O}$ arises from some maximal matrix $N \in \mathcal{O}$ by identification of some vector variables, the construction of OPBs of the $n$-qubit system reduces to the enumeration of the equivalence classes of maximal matrices $M \in \mathcal{O}$. Hence, in order to construct the OPBs of $\mathcal{H}$, it suffices to classify (up to equivalence) the maximal matrices $M \in \mathcal{O}$.

Let $A=\left[a_{i, j}\right]$ be an $s \times t$ matrix whose entries are vector variables. We say that the rows of $A$ are independent if we can assign unit vectors to these variables so that the product vectors $\left|a_{i, 1}, a_{i, 2}, \ldots, a_{i, t}\right\rangle, i=1,2, \ldots, s$, are linearly independent.

Lemma 8 If $A=\left[a_{i, j}\right]$ is an $s \times n$ matrix of vector variables whose rows are independent, then $s \leq 2^{n}$.

Proof. This follows from the fact that $\operatorname{Dim} \mathcal{H}=2^{n}$.

Example 9 Let $A \in \mathcal{O}(2)$ and let $[a b]$ be its first row. By Corollary 2, $A$ is reducible. Hence, one of the columns contains only one independent variable. By interchanging the columns if necessary, we may assume that the first column contains only one independent variable. Since $\mu(a)=\mu\left(a^{\perp}\right)$, by permuting the rows, we may assume that the first column is $\left[\begin{array}{llll}a & a & a^{\perp} & a^{\perp}\end{array}\right]^{T}$. Since the first row is orthogonal to the second, we deduce that the second row must be $\left[a b^{\perp}\right]$. There are now two choices for the remaining two entries of $A$. They lead to the two matrices $M$ and $N$ shown below. Thus, in the case of two qubits there are only two equivalence classes in $\mathcal{O}$. Their representatives are

$$
M=\left[\begin{array}{cc}
a & b \\
a & b^{\perp} \\
a^{\perp} & b \\
a^{\perp} & b^{\perp}
\end{array}\right], \quad N=\left[\begin{array}{cc}
a & b \\
a & b^{\perp} \\
a^{\perp} & c \\
a^{\perp} & c^{\perp}
\end{array}\right] .
$$

Both matrices are reducible, $M$ belongs to the standard class and $N$ is maximal. Since $M$ is obtained from $N$ by setting $c=b$, we have $M<N$. 
We shall associate to $M \in \mathcal{O}(n)$ a family $\mathcal{F}_{M}$ of OPBs of $\mathcal{H}$. To do that, we assign to all vector variables in $M$ unit vectors. It is understood that to a pair $x, x^{\perp}$ which occur in column $j$, we assign a pair of orthogonal unit vectors in $\mathcal{H}_{j}$. Denote by $\left|\alpha_{i}\right\rangle \in \mathcal{H}$ the product vector which is the tensor product of the unit vectors assigned to the entries of the row $i$ of A. Then $\left\{\left|\alpha_{i}\right\rangle: i=1,2, \ldots, 2^{n}\right\}$ is an OPB of $\mathcal{H}$. The family $\mathcal{F}_{M}$ consists of all OPBs that arise from $M$ in this way.

For instance, if $N$ is the matrix displayed above in (5) , then $\mathcal{F}_{N}$ consists of all OPBs $\left\{|x\rangle \otimes|y\rangle,|x\rangle \otimes|y\rangle^{\perp},|x\rangle^{\perp} \otimes|z\rangle,|x\rangle^{\perp} \otimes|z\rangle^{\perp}\right\}$, where $|x\rangle \in \mathcal{H}_{1}$ and $|y\rangle,|z\rangle \in \mathcal{H}_{2}$ are arbitrary unit vectors.

Note that two matrices $M, N \in \mathcal{O}$ are equivalent if and only if the corresponding families $\mathcal{F}_{M}$ and $\mathcal{F}_{N}$ are equivalent under local unitary transformations and qubit permutations. If $M$ is irreducible then, in the generic case, the members of $\mathcal{F}_{M}$ are irreducible OPBs.

If $S \in \mathcal{O}(n)$ is a standard matrix, then we say that the family $\mathcal{F}_{S}$ is the standard family. This family consists of all OPBs which are equivalent to the standard basis of $\mathcal{H}$. Given any matrix $A \in \mathcal{O}(n)$, there is a finite chain $S=A_{0}<A_{1}<\cdots<A_{m}=A, m \geq 0$, which begins with a standard matrix $S$ (on some variables) and reaches $A$. Consequently, we have $\mathcal{F}_{S} \subseteq \mathcal{F}_{A}$

One can use the OPBs in $\mathcal{F}_{M}$ to derive some properties of the matrix $M$. We illustrate this by a simple lemma.

Lemma 10 If the matrices $M, N \in \mathcal{O}(n)$ have all rows equal except possibly the first, then $M=N$.

Proof. Let us assign to all vector variables that occur in $M$ and $N$ different unit vectors. The corresponding OPBs will consist of the same vectors except possibly one of them. As they are orthonormal bases, these two OPBs must be the same. Thus the first rows of $M$ and $N$ give the same product vector. As we have assigned different unit vectors to different vector variables, we infer that the first rows of $M$ and $N$ must be the same.

It is easy to see that any $\mathrm{OPB}$ of $\mathcal{H}$ belongs to some family $\mathcal{F}_{M}, M \in \mathcal{O}$. Indeed, let $\mathcal{A}:=\left\{\left|a_{s}\right\rangle=\left|a_{s, 1}, \ldots, a_{s, n}\right\rangle: s=1, \ldots, 2^{n}\right\}$ be an OPB, and let $A$ be the $2^{n} \times n$ matrix whose entries are the unit vectors $A[s, j]=\left|a_{s, j}\right\rangle$. Note that several entries in a column $j$ of $A$ may be equal to some $\left|a_{s, j}\right\rangle$. We replace all of them by a single vector variable $v$, and likewise replace all the entries equal to $\left|a_{s, j}\right\rangle^{\perp}$ with $v^{\perp}$. The resulting matrix $M$ has vector 
variables as its entries and belongs to $\mathcal{O}$. Moreover, we have $\mathcal{A} \in \mathcal{F}_{M}$.

\section{WEAK EQUIVALENCE IN $\mathcal{O}$}

In the previous section we have reduced the problem of constructing the multiqubit OPBs to a combinatorial problem. In this section, we investigate the latter problem. We begin by introducing two more definitions. We say that a collection of rows of $M \in \mathcal{O}=\mathcal{O}(n)$ is $j$-constant if all entries of the column $j$ contained in these rows are equal to each other. For a subset $J \subseteq\{1, \ldots, n\}$ we say that a collection of rows of $M$ is $J$-compatible if these rows are $j$-constant for all $j \notin J$.

Proposition 11 Let $M \in \mathcal{O}$ be a maximal matrix, $J \subset\{1,2, \ldots, n\}$ a subset of cardinality $k>0$, and $I \subset\left\{1,2, \ldots, 2^{n}\right\}$ a subset of cardinality $2^{k}$ such that the rows of $M$ with indices in $I$ are $J$-compatible. Denote by $B$ the submatrix of $M$ contained in the intersection of rows $I$ and columns $J$. Then

(i) $B \in \mathcal{O}(k)$;

(ii) for $i \notin I$, the portion of row $i$ contained in columns $J$ is not orthogonal to all the rows of $B$;

(iii) any vector variable that occurs in $B$ does not occur in $M$ outside of $B$;

(iv) the submatrix $B$ is maximal in $\mathcal{O}(k)$;

(v) the matrix $M^{\prime}$ obtained from $M$ by permuting the columns of $B$ also belongs to $\mathcal{O}$ and it is maximal.

Proof. (i) Since the rows $I$ of $M$ are $J$-compatible, we deduce that all rows of $B$ must be mutually orthogonal and so $B \in \mathcal{O}(k)$.

(ii) This follows from Lemma 8 .

(iii) Assume that a vector variable, say $a$, occurs in $B$ and also outside $B$. Let $N$ be the matrix obtained from $M$ by replacing each ocurrence of $a$ and $a^{\perp}$ inside $B$ by a new vector variable and its perpendicular, respectively.

We claim that $N \in \mathcal{O}$. We have to verify that $N$ satisfies the conditions (i-iii) of $\mathcal{O}$. The conditions (i) and (ii) obviously hold. In order to verify the condition (iii) it suffices to show that if $m \notin I$ then the row $m$ of $M$ is orthogonal to all rows of $N$ in $I$. By part (ii) there exists $i \in I$ such that the portion of row $m$ in $J$ is not orthogonal to the row $i$ of $B$. Hence, 
the portion of row $m$ outside $J$ must be orthogonal to the corresponding portion of the row $i$ of $M$. Since the rows $I$ of $M$ are $J$-compatible, we conclude that row $m$ is orthogonal to all rows of $N$ in $I$. Thus our claim is proved. Obviously we have $M<N$, which contradicts the hypothesis that $M$ is maximal.

(iv) follows from (iii) and the maximality of $A$.

(v) follows immediately from the previous assertions.

We refer to the operation $M \rightarrow M^{\prime}$ described in the above proposition as a switching operation. Let us give an example.

Assume that a maximal matrix $M \in \mathcal{O}$ has a $4 \times 2$ submatrix $X$ contained in columns $k$ and $l$ such that the four rows containing this submatrix are $\{k, l\}$-compatible. Then $X \in \mathcal{O}(2)$ and, by using a switching operation, we can replace the submatrix $X$ by the matrix $Y \in \mathcal{O}(2)$

$$
X=\left[\begin{array}{cc}
a & x \\
a & x^{\perp} \\
a^{\perp} & y \\
a^{\perp} & y^{\perp}
\end{array}\right] \rightarrow Y=\left[\begin{array}{cc}
x & a \\
x^{\perp} & a \\
y & a^{\perp} \\
y^{\perp} & a^{\perp}
\end{array}\right]
$$

to obtain another maximal matrix $M^{\prime} \in \mathcal{O}$.

Definition 12 We say that two maximal matrices $M, N \in \mathcal{O}(n)$ are weakly equivalent if there is a finite sequence $M=M_{0}, M_{1}, \ldots, M_{k}=N$ in $\mathcal{O}$ such that each arrow $M_{i-1} \rightarrow M_{i}$ is an equivalence or switching operation. We shall refer to the equivalence classes of the weak equivalence relation as switching classes.

Each switching class in $\mathcal{O}$ consists of maximal matrices and it is a disjoint union of finitely many previously defined equivalence classes. The construction of matrices in $\mathcal{O}(n)$ can be carried out in two steps: first find the representatives of the switching classes, and then find the representatives of the equivalence classes contained in each switching class.

By the above definition, two equivalent maximal matrices are also weakly equivalent. The converse is false. For example the reducible maximal matrices of three qubits form a single switching class which is the union of two equivalence classes (see Lemma 14 below). It follows from Proposition 11 (iii) that a switching class cannot contain a reducible and a irreducible matrix. 
When displaying matrices $M$ we shall use some shorthand notation in order to diminish the number of rows. It is also convenient to specify one of the vector variables in some column to be the standard basis vector $|0\rangle$. For instance, if $a$ occurs in column $j$ then we can replace in that column each $a$ with 0 and each $a^{\perp}$ with 1 . This reduces the number of vector variables by one. We say that the column $j$ of the resulting matrix is normalized. Note that this normalization is not unique.

We often simplify a maximal matrix $M$ by using the symbol *. Assume that a vector variable, say $a$, occurs in row $i$ and column $k$ of $M$. Then $a^{\perp}$ also occurs in column $k$ and, say, row $j$. Assume also that the rows $i$ and $j$ are $\{k\}$-compatible. Since $M$ is maximal, we must have $\mu(a)=1$ by Proposition 11 (iii). Under these assumptions we can replace $a$ in row $i$ with $*$ and delete row $j$. We can recover (up to ordering of the rows and naming of the vector variables) the original $M$ from this simplified matrix by reversing this procedure. For instance, if $n=4$ then the symbolic row $[* b c d]$ is a shorthand for the pair of rows

$$
\left[\begin{array}{cccc}
a & b & c & d \\
a^{\perp} & b & c & d
\end{array}\right] .
$$

For a concrete example see (8) where we simplified the matrix $M_{\text {nor }}$ to get a matrix with 5 rows only.

We may apply this simplification several times one after the other. For instance, when $n=4$ the two rows

$$
\left[\begin{array}{llll}
* & a & b & c \\
* & a & b & c^{\perp}
\end{array}\right] .
$$

stand for the following four rows

$$
\left[\begin{array}{cccc}
d & a & b & c \\
d^{\perp} & a & b & c \\
e & a & b & c^{\perp} \\
e^{\perp} & a & b & c^{\perp}
\end{array}\right]
$$

where $d$ and $e$ are distinct two new independent vector variables.

Let us give two small examples.

Example 13 First, in the case of two qubits there is only one maximal matrix $N \in \mathcal{O}$ up 
to equivalence. This is the matrix shown in (5). Its normalized version is

$$
\left[\begin{array}{rr}
0 & 0 \\
1 & 0 \\
b & 1 \\
b^{\perp} & 1
\end{array}\right]
$$

where we have specified that $|a\rangle=|0\rangle$ and $|c\rangle=|0\rangle$. Note that $M$ is reducible since its second column contains only one independent vector variable.

Second, according to [9] there is a unique family of irreducible three-qubit OPBs. In our notation, this family is given by the following matrix $M$

$$
M=\left[\begin{array}{ccc}
u & v & w \\
a & v^{\perp} & w \\
a^{\perp} & v^{\perp} & w \\
u & b & w^{\perp} \\
u & b^{\perp} & w^{\perp} \\
u^{\perp} & v & c \\
u^{\perp} & v & c^{\perp} \\
u^{\perp} & v^{\perp} & w^{\perp}
\end{array}\right], \quad M_{\mathrm{nor}}=\left[\begin{array}{ccc}
0 & 0 & 0 \\
a & 1 & 0 \\
a^{\perp} & 1 & 0 \\
0 & b & 1 \\
0 & b^{\perp} & 1 \\
1 & 0 & c \\
1 & 0 & c^{\perp} \\
1 & 1 & 1
\end{array}\right], \quad\left[\begin{array}{ccc}
0 & 0 & 0 \\
* & 1 & 0 \\
0 & * & 1 \\
1 & 0 & * \\
1 & 1 & 1
\end{array}\right]
$$

The matrix $M_{\text {nor }}$ is the normalization of $M$.

Apart from the asterisks and various vector variables $a$ and their perpendiculars $a^{\perp}$, all other entries of $M$ (when displayed) are the standard basis vectors $|0\rangle$ and $|1\rangle$ of the $\mathcal{H}_{i}$. (These standard basis vectors are introduced by the normalization process mentioned above.)

Let us now consider the case $n=3$.

Lemma 14 In $\mathcal{O}(3)$ there are only two switching classes of maximal matrices: one of them consists of irreducible and the other of reducible matrices. The former is a single equivalence classcas with representative (8) while the latter splits into two equivalence classes with 
representatives

$$
\left[\begin{array}{ccc}
a & b & c \\
a & b & c^{\perp} \\
a & b^{\perp} & d \\
a & b^{\perp} & d^{\perp} \\
a^{\perp} & e & f \\
a^{\perp} & e & f^{\perp} \\
a^{\perp} & e^{\perp} & g \\
a^{\perp} & e^{\perp} & g^{\perp}
\end{array}\right], \quad\left[\begin{array}{ccc}
a & b & c \\
a & b & c^{\perp} \\
a & b^{\perp} & d \\
a & b^{\perp} & d^{\perp} \\
a^{\perp} & f & e \\
a^{\perp} & f^{\perp} & e \\
a^{\perp} & g & e^{\perp} \\
a^{\perp} & g^{\perp} & e^{\perp}
\end{array}\right]
$$

Proof. In view of the Example [13, it suffices to consider the case when $M \in \mathcal{O}(3)$ is reducible. We may assume that the first column of $M$ contains a vector variable $a$ with multiplicity $\mu(a)=4$. After permuting the rows, we may assume that

$$
M=\left[\begin{array}{r|r}
a & \\
a & M^{\prime} \\
a & \\
a & \\
\hline a^{\perp} & \\
a^{\perp} & M^{\prime \prime} \\
a^{\perp} & \\
a^{\perp} &
\end{array}\right] .
$$

Since $M$ is maximal, Proposition 11 (i) implies that $M^{\prime}, M^{\prime \prime} \in \mathcal{O}(2)$. Moreover these two submatrices have no vector variable in common. By using Example 13, it is now easy to verify that there are only two possibilities (up to equivalence) as stated in the lemma.

Let us introduce additional notation and invariants which will be used when testing whether two matrices in $\mathcal{O}(n)$ are equivalent.

Given a matrix $M \in \mathcal{O}(n)$, we denote by $\nu_{i}$ the number of independent vector variables which occur in column $i$ of $M$. Assume that these variables are $a_{j}, j=1,2, \ldots, \nu_{i}$, and set $\mu_{i, j}=\mu\left(a_{j}\right)$. We assume that the indexing is chosen so that $\mu_{i, j} \geq \mu_{i, k}$ for $j<k$, and we set $\mu_{i}=\mu_{i, 1}$. The numbers $\mu_{i, 1}, \mu_{i, 2}, \ldots, \mu_{i, \nu_{i}}$ form a partition $\pi_{i}$ of the integer $2^{n-1}$. We shall order these partitions in the decreasing lexicographic order. 


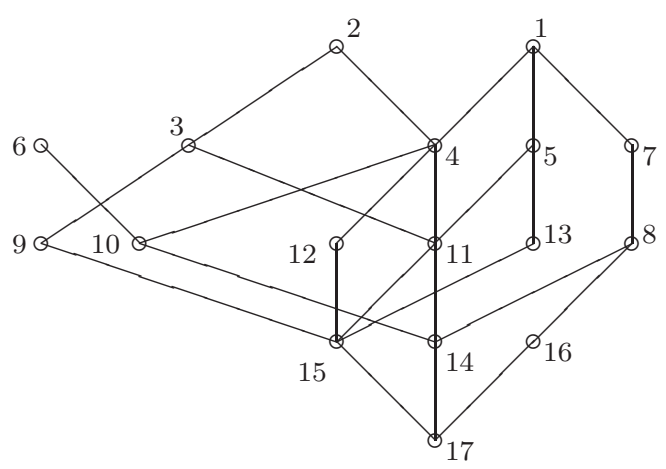

$$
\begin{aligned}
& \nu=7 \\
& \nu=6 \\
& \nu=5 \\
& \nu=4 \\
& \nu=3
\end{aligned}
$$

Figure 1. Hasse diagram of the partially ordered set of equivalence classes in $\mathcal{O}(3)$. The partial order is induced by the relation " $<$ ".

By permuting the columns of $M$, we may assume that $\pi_{1} \geq \pi_{2} \geq \cdots \geq \pi_{n}$. In particular $\mu_{i} \geq \mu_{j}$ for $i<j$.

Another important invariant of matrices $M \in \mathcal{O}(n)$ is the total number $\nu_{M}=\sum \nu_{i}$ of independent vector variables that occur in $M$. The dimension of the family $\mathcal{F}_{M}$ is equal to $2 \nu_{M}$ because each of the vector variables makes the contribution of 2 to this dimension.

By identifying two independent varables in a single column of one of the three maximal matrices (8) and (9), and by repeating this procedure as far as possible we obatin a bunch of matrices in $\mathcal{O}(3)$. By selecting a maximal subset of pairwise nonequivalent matrices in this bunch, we obtain the following corollary. The Hasse diagram of the equivalence classes of $\mathcal{O}(3)$, for the partial order induced by the relation " $<$ ", is shown on Fig. 1.

Corollary 15 There are 17 equivalence classes in $\mathcal{O}(3)$. Their representatives $M$ are listed below together with associated partitions $\pi_{1}, \pi_{2}, \pi_{3}$ and the parameter $\nu_{M}$. (All columns but one are normalized. On Fig. 1, the equivalence classes are numbered according to their position in the list.) 


$$
\begin{aligned}
& {\left[\begin{array}{lll}
0 & 0 & * \\
0 & 1 & * \\
1 & y & * \\
1 & y^{\perp} & *
\end{array}\right] \begin{array}{l}
2^{2} \\
\nu=7
\end{array}\left[\begin{array}{lll}
0 & 0 & * \\
0 & 1 & * \\
1 & * & 0 \\
1 & * & 1
\end{array}\right] \begin{array}{l}
4,1^{2} \\
2,1^{2} \\
\nu=7
\end{array}\left[\begin{array}{lll}
0 & 0 & * \\
0 & 1 & * \\
1 & * & 0 \\
1 & 0 & 1 \\
1 & 1 & 1
\end{array}\right] \begin{array}{l}
4,1 \\
\nu=6
\end{array}}
\end{aligned}
$$

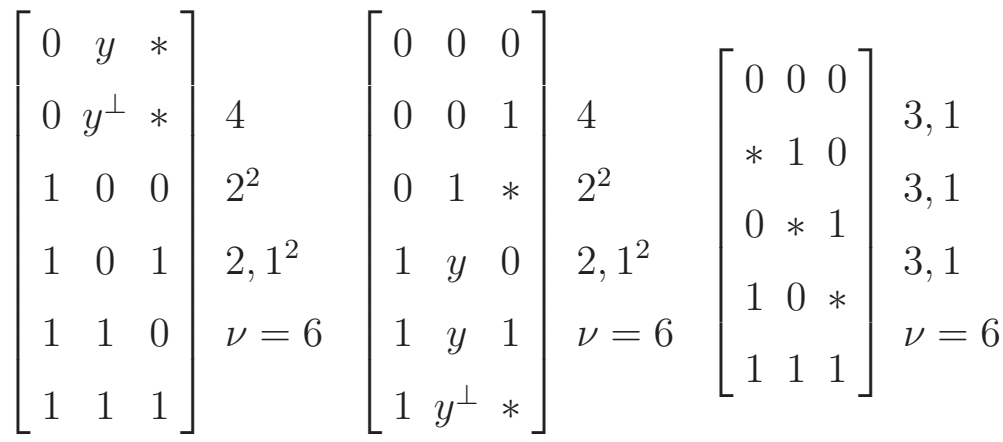

$$
\begin{aligned}
& \left.\left.\left[\begin{array}{lll}
0 & 0 & * \\
0 & 1 & * \\
1 & 0 & * \\
1 & 1 & *
\end{array}\right] \begin{array}{l}
4 \\
1^{4} \\
\nu
\end{array}\left[\begin{array}{lll}
0 & 0 & 0 \\
0 & 0 & 1 \\
0 & 1 & * \\
1 & 0 & * \\
1 & 1 & 0 \\
1 & 1 & 1
\end{array}\right] \begin{array}{l}
2,1^{2} \\
\nu=5 \\
1
\end{array}\right] \begin{array}{lll}
0 & 0 & * \\
0 & 1 & * \\
1 & 0 & 0 \\
1 & 0 & 1 \\
1 & 1 & 0
\end{array}\right] \begin{array}{l}
4 \\
2,1^{2} \\
\nu=5
\end{array}
\end{aligned}
$$

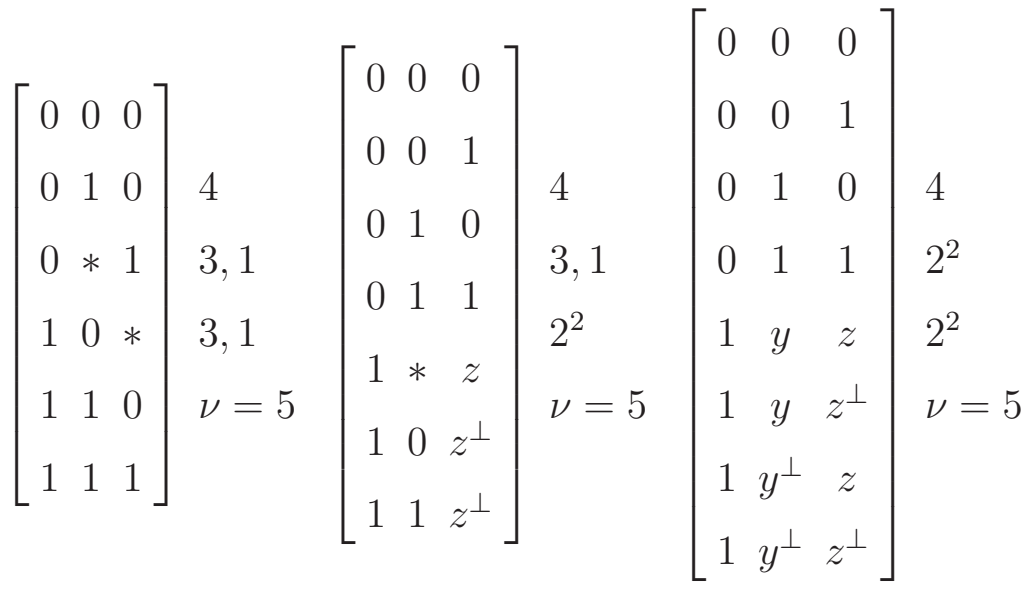




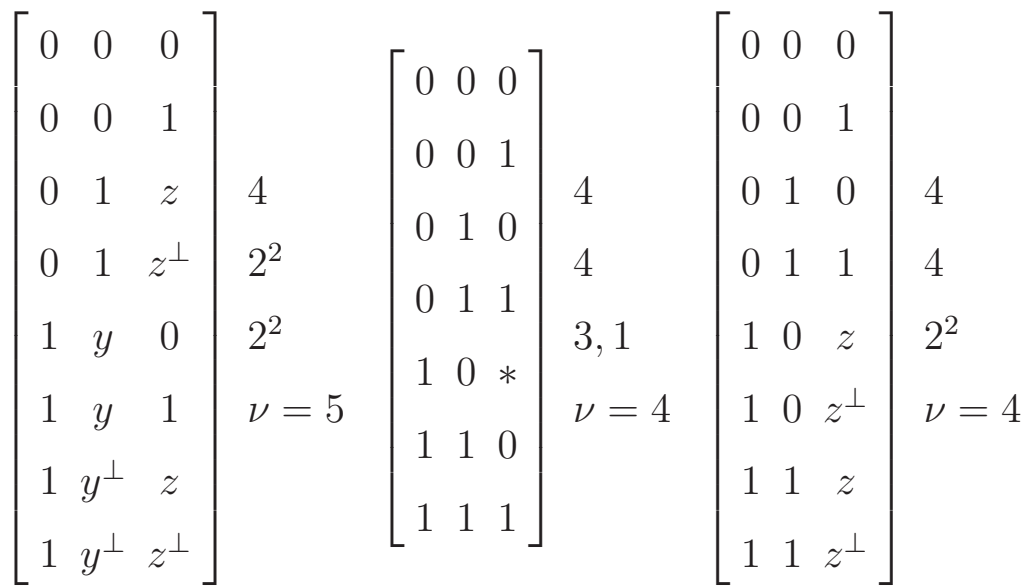

$$
\begin{aligned}
& {\left[\begin{array}{lll}
0 & 0 & 0 \\
0 & 0 & 1 \\
0 & 1 & z \\
0 & 1 & z^{\perp} \\
1 & 0 & z \\
1 & 0 & z^{\perp} \\
1 & 1 & 0 \\
1 & 1 & 1
\end{array}\right] \quad \nu=4\left[\begin{array}{lll}
0 & 0 & 0 \\
0 & 0 & 1 \\
0 & 1 & 0 \\
0 & 1 & 1 \\
1 & 0 & 0 \\
1 & 0 & 1 \\
1 & 1 & 0 \\
1 & 1 & 1
\end{array}\right] \begin{array}{l}
4 \\
4
\end{array}}
\end{aligned}
$$

\section{CLASSIFICATION OF FOUR-QUBIT ORTHOGONAL PRODUCT BASES}

In this section we classify the four-qubit OPBs by using the weak equivalence defined in the previous section. More precisely, we solve our combinatorial problem in the case of four-qubits proposed in Sec. III. We obtain 33 equivalence classes of matrices in $\mathcal{O}(4)$ and list the representatives $A$ of these classes. The corresponding 33 families $\mathcal{F}_{A}$ cover all OPBs up to equivalence. Equivalently, each $\mathrm{OPB}$ is equivalent to one belonging to these 33 families. However, for a given $\mathrm{OPB}$, such family $\mathcal{F}_{A}$ does not have to be unique. To obtain uniqueness, one has to replace the families $\mathcal{F}_{A}$ by somewhat smaller families which we denote by $\mathcal{F}_{A}^{\#}$ and call strict families. For this see section VII. 


\section{A. Preliminaries}

We introduce two important lemmas. They will be used in proving the main result of this paper, Theorem 19.

Lemma 16 Let $A=\left[a_{i, j}\right] \in \mathcal{O}(3)$ and assume that $a_{1, j}=a_{2, j}$ holds true for at most one $j \in\{1,2,3\}$. If a row of vector variables $r=\left[\begin{array}{lll}u & v w\end{array}\right]$ is orthogonal to the last 6 rows of $A$, then $r$ is equal to the first or second row of $A$.

Proof. Let us assign to all vector variables (including $u, v$ and $w$ ) unit vectors such that different vector varables are assigned different unit vectors. It is understood that to a pair $x, x^{\perp}$ we assign a pair of orthogonal unit vectors. Let $\left|\alpha_{i}\right\rangle$ be the product vector obtained from the row $i$ of $A$, and $|\xi\rangle$ the product vector obtained from $r$. The hypothesis implies that the 2-dimensional subspace spanned by $\left|\alpha_{1}\right\rangle$ and $\left|\alpha_{2}\right\rangle$ contains no other product vectors (up to scalar multiple). As $|\xi\rangle$ belongs to this subspace, we must have $|\xi\rangle=\left|\alpha_{1}\right\rangle$ or $|\xi\rangle=\left|\alpha_{2}\right\rangle$. We conclude that $r$ must be equal to the first or second row of $A$.

By inspection of the matrix (8), it is easy to see that the following corollary holds.

Corollary 17 Let $A \in \mathcal{O}(3)$ be irreducible and let $\left[\begin{array}{lll}a & b & c\end{array}\right]$ and $\left[\begin{array}{lll}x & y & z\end{array}\right]$ be two of its rows. Then the following assertions hold:

(i) each entry has multiplicity 1 or 3;

(ii) if two of the equalities $a=x^{\perp}, b=y^{\perp}, c=z^{\perp}$ hold, then all of them hold;

(iii) if $a=x^{\perp}$ and $\mu(a)=1$ then $b=y$ and $c=z$.

These simple facts will be used many times in the proofs below and in the next section.

For convenience we introduce some additional notation. For any matrix $A \in \mathcal{O}(4)$ and index sequences $i_{1}<\cdots<i_{s}$ and $j_{1}<\cdots<j_{t}$ we denote by $A\left[i_{1}, \ldots, i_{s} ; j_{1}, \ldots, j_{t}\right]$ the $s \times t$ submatrix of $A$ contained in rows $i_{1}, \ldots, i_{s}$ and columns $j_{1}, \ldots, j_{t}$.

Let $\nu_{1}=\nu_{1}(A)$, the maximal number of independent vector variables in the first column of $A$. We select $\nu_{1}$ independent varables $\xi_{1}, \xi_{2}, \ldots, \xi_{\nu_{1}}$ from this column and arrange them so that their multiplicities $\mu_{1, i}$ weakly decrease, i.e., $\mu_{1,1} \geq \mu_{1,2} \geq \cdots \geq \mu_{1, \nu_{1}}$. After permuting the rows of $A$, we may assume that its first column consists of $\mu_{1,1}$ entries $\xi_{1}$, followed by $\mu_{1,2}$ entries $\xi_{2}, \ldots$, then $\mu_{1,1}$ entries $\xi_{1}^{\perp}$, followed by $\mu_{1,2}$ entries $\xi_{2}^{\perp}, \ldots$. Let us partition $A$ horizontally into $2 \nu_{1}$ blocks $\tilde{N}_{i}, i=0,1, \ldots, 2 \nu_{1}-1$ such that the elements in the first 
column of $\tilde{N}_{i}$ are all equal to $\xi_{i+1}$ if $i<\nu_{1}$ and are equal to $\xi_{i-\nu_{1}+1}^{\perp}$ if $i \geq \nu_{1}$. We let $N_{i}$ be the matrix obtained from $\tilde{N}_{i}$ by deleting the first column.

Finally we define $M_{i}$ to be the matrix

$$
M_{i}:=\left[\begin{array}{c}
N_{i} \\
N_{i+1} \\
\vdots \\
N_{i+\nu_{1}-1}
\end{array}\right]
$$

where the indices are to be reduced modulo $2 \nu_{1}$. Note that the rows of $M_{i}$ are mutually orthogonal, and so each of the matrices $M_{i}$ belongs to $\mathcal{O}(3)$.

In the case $\nu_{1}=1$ we just have $M_{0}=N_{0}$ and $M_{1}=N_{1}$. Moreover, if $A$ is maximal then $M_{0}$ and $M_{1}$ are maximal and they have no vector variable in common.

In the next lemma we investigate the maximum of multiplicities of entries in the matrices of $\mathcal{O}(4)$. The symbols $M_{i}, N_{i}$ have been introduced above and we recall that $\mu_{i}=\mu_{i, 1}$ is the largest of the $\mu_{i, j}$.

Lemma 18 If $A:=\left[a_{s, i}\right] \in \mathcal{O}(4)$ and $\mu_{1} \geq \mu_{2}, \mu_{3}, \mu_{4}$ then $\mu_{1} \geq 6$.

Proof. If $B<A$ it is immediate from the definition of ";" that $\mu_{1}(A) \leq \mu_{1}(B)$. It follows that $\mu_{1}$ attains its minimum at a maximal matrix. Hence, without any loss of generality we can assume that $A$ is maximal.

By Proposition 1(iii) we have $\sum_{i=1}^{4} \mu\left(a_{s, i}\right) \geq 15$ for each $s \in\{1,2, \ldots, 16\}$. Hence $\mu_{1} \geq 4$.

Suppose that $\mu_{1}=4$. Then $\mu(x) \in\{3,4\}$ for each entry $x$ of $A$. Since the multiplicities of any maximal set of independent vector variables of any column must add up to 8, we conclude that $\mu(x)=4$ for each entry $x$ of $A$. Thus each column of $A$ contains exactly two independent vector variables. By permuting the rows of $A$, we may assume that the first

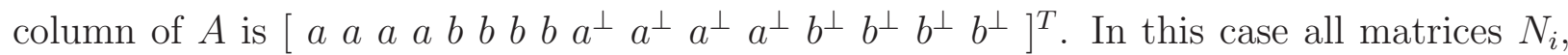
$i=0,1,2,3$, have size $4 \times 3$.

Suppose that at least one of the $M_{i}$, say $M_{0}$, is reducible. Thus one of the columns of $M_{0}$ contains only one independent variable, say $c$. By permuting the columns 2,3,4 of $A$ we may assume that the first column of $M_{0}$ has four of its entries equal to $c$ and the remaining four equal to $c^{\perp}$. As $\mu(x)=4$ for all $x$, the first column of $M_{2}$ has four entries equal to $d$ and four equal to $d^{\perp}\left(d\right.$ another vector variable). Hence, $M_{2}$ is reducible. Since $M_{1} \in \mathcal{O}(3)$, exactly 
2 entries of $N_{1}$ are equal to $c$, and exactly 2 entries of $N_{2}$ are equal to $d$. Consequently, we may assume that the second column of $A$ is $\left[c c c^{\perp} c^{\perp} c c c^{\perp} c^{\perp} d d d^{\perp} d^{\perp} d d d^{\perp} d^{\perp}\right]^{T}$. Let us partition the $16 \times 2$ submatrix of $A$, consisting of the last two columns, into eight $2 \times 2$ blocks $L_{i}, i=0,1, \ldots, 7$. Note that the eight submatrices $K_{i}:=\left[\begin{array}{c}L_{i} \\ L_{i+2}\end{array}\right], i=0,1, \ldots, 7$ (indices are modulo 8) belong to $\mathcal{O}(2)$. Moreover, the matrix $K^{\prime}:=\left[\begin{array}{c}L_{3} \\ L_{4}\end{array}\right]$ also belongs to $\mathcal{O}(2)$.

Assume that one of the columns of some $L_{i}$ has two equal entries, say $x:=a_{1,3}=a_{2,3}$. Since $K_{0} \in \mathcal{O}(2)$ we must have $a_{5,3}=a_{6,3}=x^{\perp}$. Similarly, since $K_{2}, K_{4} \in \mathcal{O}(2)$, we deduce that $a_{9,3}=a_{10,3}=x$ and $a_{13,3}=a_{14,3}=x^{\perp}$. Since $K^{\prime} \in \mathcal{O}(2)$, we obtain that $a_{7,3}=a_{8,3}=x^{\perp}$. This is impossible since $\mu\left(x^{\perp}\right)=4$. We conclude that no column of any $L_{i}$ consists of two equal entries.

Since the rows of $L_{0}$ are orthogonal, one of its columns, say the first, has the form $\left[x x^{\perp}\right]^{T}$. Recall that each matrix in $\mathcal{O}(2)$ is equivalent to one of the two matrices in (5) $)$. By inspection of these two matrices and by taking into account that the two entries of the second column of $L_{0}$ are not equal, we deduce that the first column of $L_{2}$ must consist of $x$ and $x^{\perp}$. By repeating this argument, it follows that the entries of the first column of $L_{i}$ for $i$ even are $x$ and $x^{\perp}$. As $K^{\prime} \in \mathcal{O}(2)$, the same is true for $L_{3}$. This is impossible since $\mu(x)=4$. We conclude that all $M_{i}$ are irreducible.

As $M_{0}$ is irreducible, there are independent vector variables $x, y, z, u, v, w$ such that $x, y, z$ have multiplicity 3 in $M_{0}$ and $u, v, w$ multiplicity 1 in $M_{0}$, with $x, u$ in the first column, $y, v$ in the second and $z, w$ in the third column of $M_{0}$. Consequently, $x, y, z$ have multiplicity 1 in $M_{2}$ and $u, v, w$ multiplicity 3 in $M_{2}$. As $M_{2}$ is also irreducible, by using Example 13, we 
infer that there exist permutation matrices $P$ and $Q$ such that

$$
M_{0}=P\left[\begin{array}{ccc}
x & y & z \\
u & y^{\perp} & z \\
u^{\perp} & y^{\perp} & z \\
x & v & z^{\perp} \\
x & v^{\perp} & z^{\perp} \\
x^{\perp} & y & w \\
x^{\perp} & y & w^{\perp} \\
x^{\perp} & y^{\perp} & z^{\perp}
\end{array}\right], \quad M_{2}=Q\left[\begin{array}{ccc}
u & v & w \\
x & v^{\perp} & w \\
x^{\perp} & v^{\perp} & w \\
u & y & w^{\perp} \\
u & y^{\perp} & w^{\perp} \\
u^{\perp} & v & z \\
u^{\perp} & v & z^{\perp} \\
u^{\perp} & v^{\perp} & w^{\perp}
\end{array}\right] .
$$

Let $R:=\left[s^{\perp} u v w\right], s \in\{a, b\}$, be the row of $A$ containing the row $[u v w]$ of $M_{2}$. Let $t \in\{a, b\}$ be different from $s$. Since $[u v w]$ is orthogonal to only three rows of $M_{0}, R$ is not orthogonal to at least one of the four rows of $A$ whose first element is $t$. Thus we have a contradiction. We conclude that $\mu_{1} \geq 5$.

Suppose that $\mu_{1}=5$. There are three possibilities for the partition $\pi_{1}$ associated to the first column of $A$. In each of these cases we shall obtain a contradiction.

Case 1: $\pi_{1}=5,3$.

We may assume that $\left[\text { a } a \text { a } a \text { a } b b b a^{\perp} a^{\perp} a^{\perp} a^{\perp} a^{\perp} b^{\perp} b^{\perp} b^{\perp}\right]^{T}$ is the first column of $A$. In this case $N_{0}$ and $N_{2}$ have the size $5 \times 3$, while $N_{1}$ and $N_{3}$ have size $3 \times 3$. Assume that one of the columns of $N_{1}$, say the first column, consists of 3 equal entries $x=a_{6,2}=a_{7,2}=a_{8,2}$. Since $M_{0} \in \mathcal{O}(3)$, the first column of $N_{0}$ must contain at least 3 entries $x^{\perp}$. Similarly, the first column of $N_{2}$ must contain at least 3 entries $x^{\perp}$. This contradicts the inequality $\mu\left(x^{\perp}\right) \leq 5$. We conclude that no column of $N_{1}$ consists of 3 equal entries.

Subcase 1a: At least one of the $M_{i}$, say $M_{0}$, is reducible.

We may assume that $a_{i, 2} \in\left\{c, c^{\perp}\right\}$ for $1 \leq i \leq 8$. As the three entries of the first column of $N_{1}$ are not equal, we may assume that the first column of $M_{0}$ is $\left[c^{\perp} c^{\perp} c^{\perp} c c c c c^{\perp}\right]^{T}$. Since $M_{3} \in \mathcal{O}(3)$, one of the entries in the first column of $N_{3}$ must be $c$. As $\mu_{1}=5$ we must have $\mu(c)=5$. Hence, we may assume that the first column of $N_{3}$ is [c $\left.d d^{\perp}\right]$, where $d \notin\left\{c, c^{\perp}\right\}$. Note that $c$ has multiplicity 2 in $M_{1}$. By Corollary 17 (i), $M_{1}$ is reducible. Thus, we may assume that $a_{i, 3} \in\left\{e, e^{\perp}\right\}$ for $6 \leq i \leq 13$, where $e$ is a new vector variable. Since the entries in the second column of $N_{1}$ cannot be the same, we may assume that the second column of $N_{1}$ has two entries $e$ and one entry $e^{\perp}$. Since the rows 4,5,6,7 of $A$ are orthogonal to each other, we must have $A[4,5,6,7 ; 3,4] \in \mathcal{O}(2)$. The first two entries of the second 
column of $N_{1}$ cannot be both equal to $e$. Indeed, this would imply that $a_{4,3}=a_{5,3}=e^{\perp}$ which contradicts the fact that $e^{\perp}$ has multiplicity 4 in $M_{1} \in \mathcal{O}(3)$. Consequently, we may assume that the second column of $N_{1}$ is $\left[e e^{\perp} e\right]^{T}$. Since $M_{0} \in \mathcal{O}(3)$ and $\mu(e) \leq 5$, we infer that $e^{\perp}$ occurs exactly once in $N_{0}$. Similarly, e occurs exactly once in $N_{3}$. We may assume that it does not occur in the last row of $A$. The first 3 entries of this row have multiplicity at most 3. Hence the last entry must have multiplicity at least 6 by Proposition 10 (iii). We have a contradiction with the assumption $\mu_{1}=5$.

Subcase 1b: Each $M_{i}$ is irreducible.

Thus $M_{0}$ is equivalent to the matrix (8). Suppose that the entries of $N_{1}$ are pairwise distinct. It is easy to check that any $3 \times 3$ submatrix of the matrix (8), which has pairwise distinct entries, cannot include the first or last row and must contain only one row of each pair of rows $\{2,3\},\{4,5\},\{6,7\}$. Hence, we may assume that

$$
N_{1}=\left[\begin{array}{ccc}
u & y & z \\
x & v & z^{\perp} \\
x^{\perp} & y^{\perp} & w
\end{array}\right] .
$$

The entries $u, v, w$ in $N_{1}$ correspond to $a$ or $a^{\perp}, b$ or $b^{\perp}, c$ or $c^{\perp}$ in the matrix (8)), respectively. Hence, each of $x, y, z$ must have multiplicity 3 in $M_{0}$. Similarly, they also have multiplicity 3 in $M_{1}$. Since $M_{3}$ is irreducible, the first column of $N_{3}$ must contain the elements $u, x, x^{\perp}$. Thus $x$ occurs 2 times in both $N_{0}$ and $N_{2}$ and once in both $N_{1}$ and $N_{3}$. This contradicts the inequality $\mu(x) \leq 5$. We conclude that at least one of the columns of $N_{1}$ must contain two equal entries.

We may assume that $c:=a_{6,2}=a_{7,2}$. Since $M_{0}$ and $M_{1}$ are irreducible, the multiplicity of $c$ in $M_{0}$ and $M_{1}$ must be 3 . If $a_{8,2} \neq c^{\perp}$ then both $N_{0}$ and $N_{2}$ would contain 3 entries $c^{\perp}$, contradicting the inequality $\mu(c) \leq 5$. Thus we must have $a_{8,2}=c^{\perp}$. It follows that both $N_{0}$ and $N_{2}$ contain exactly two entries equal to $c^{\perp}$ and only one entry equal to $c$. Since $M_{2}$ is irreducible, the multiplicity of $c^{\perp}$ in $M_{2}$ must be 3 . It follows that the first column of $N_{3}$ has two entries equal to $c$. This contradicts the inequality $\mu(c) \leq 5$.

Case 2: $\pi_{1}=5,2,1$.

We may assume that [ $a$ a $a$ a $a$ a $\left.b \quad b c c a^{\perp} a^{\perp} a^{\perp} a^{\perp} a^{\perp} b^{\perp} b^{\perp} c^{\perp}\right]^{T}$ is the first column of A. Since both $M_{0}, M_{5} \in \mathcal{O}(3)$, Lemma 10 implies that $N_{2}=N_{5}$. Let $N_{2}=\left[\begin{array}{ll}x & z\end{array}\right]$. As $\mu(c)=1$, Proposition 1 (iii) implies that $\mu(x)+\mu(y)+\mu(z) \geq 14$. We may assume that 
$\mu(x)=\mu(y) 5$ and $\mu(z)=4$. Thus $x$ must have multiplicity 2 or 4 in either $M_{0}$ or $M_{3}$. By Corollary 17 (i), either $M_{0}$ or $M_{3}$ is reducible. We may assume that $M_{0}$ is reducible. Since $\mu(z)=4$ and $a_{8,4}=a_{16,4}=z$, either $x$ or $y$ must have multiplicity 4 in $M_{0}$. We may assume that $x$ has multiplicity 4 in $M_{0}$. Then $x$ occurs only once in $M_{3}$, and so $x$ occurs in neither $N_{3}$ nor $N_{4}$. Since $M_{1} \in \mathcal{O}(3)$ and $a_{6,2}, a_{7,2} \in\left\{x, x^{\perp}\right\}$, the multiplicity of $x$ in $M_{1}$ must be 2 . Moreover, $a_{6,2} \neq a_{7,2}$. Indeed, $a_{6,2}=a_{7,2}=x$ contradicts the fact that $x$ has multiplicity 2 in $M_{1}$ and $a_{6,2}=a_{7,2}=x^{\perp}$ contradicts the fact that $x$ does not occur in $N_{3}$. Furthermore we cannot have $a_{6,3}=a_{7,3}$ and $a_{6,4}=a_{7,4}$ since $A$ is maximal and the submatrix $A[6,7 ; 2]$ would contradict Proposition 11 (iii). Hence we can now apply Lemma 16 to $M_{1}$ and the two rows of $N_{4}$. We deduce that the two entries in the first column of $N_{4}$ must belong to $\left\{x, x^{\perp}\right\}$. This contradicts the fact that $\mu(x)=5$.

Case 3: $\pi_{1}=5,1^{3}$. We may assume that [ a a a a a $\left.\quad b \quad c \quad d \quad a^{\perp} a^{\perp} a^{\perp} a^{\perp} a^{\perp} b^{\perp} c^{\perp} d^{\perp}\right]^{T}$ is the first column of $A$. Since both $M_{0}, M_{7} \in \mathcal{O}(3)$, Lemma 10 implies that $N_{3}=N_{7}$. Let $N_{3}=\left[\begin{array}{lll}x & y & z\end{array}\right]$. Similarly, we can show that $N_{2}=N_{6}$ and $N_{1}=N_{5}$. As in the previous case, we can show that $M_{0}$ or $M_{4}$ is reducible. We may assume that $M_{0}$ is reducible, and that $x$ has multiplicity 4 in $M_{0}$. Since $N_{i}=N_{i+4}$ for $i=1,2,3$, we must have $\mu(x)>5$ or $\mu\left(x^{\perp}\right)>5$. Thus we have a contradiction.

This completes the proof.

\section{B. Main result}

Let us now state our main result.

Theorem 19 The maximal matrices in $\mathcal{O}(4)$ split into 33 equivalence classes. They are grouped into 15 switching classes whose representatives are listed below (some columns are normalized). When a switching class contains more than one equivalence class, we list their representatives in the appendix. 
$\left.\left.\left[\begin{array}{cccc}0 & 0 & 0 & * \\ 0 & 0 & 1 & * \\ 0 & 1 & c & * \\ 0 & 1 & c^{\perp} & * \\ 1 & b & x & * \\ 1 & b & x^{\perp} & * \\ 1 & b^{\perp} & y & * \\ 1 & b^{\perp} & y^{\perp} & *\end{array}\right]+\left[\begin{array}{cccc}0 & 0 & c & * \\ 0 & 0 & c^{\perp} & * \\ 0 & 1 & x & * \\ 0 & 1 & x^{\perp} & * \\ 1 & b & 0 & 0 \\ 1 & * & 1 & 0 \\ 1 & b & * & 1 \\ 1 & b^{\perp} & 0 & * \\ 1 & b^{\perp} & 1 & 1\end{array}\right] \begin{array}{l}3,3,1 \\ 3,2^{2}, 1 \\ 3,1^{5} \\ 0\end{array}\right] \begin{array}{cccc}0 & 0 & 0 & * \\ 0 & b & 1 & * \\ 0 & b^{\perp} & 1 & * \\ 1 & 0 & c & * \\ 1 & 0 & c^{\perp} & * \\ 1 & 1 & 1 & * \\ a & 1 & 0 & * \\ a^{\perp} & 1 & 0 & *\end{array}\right] \begin{aligned} & 6,2 \\ & 1^{8} \\ & \\ & \end{aligned}$

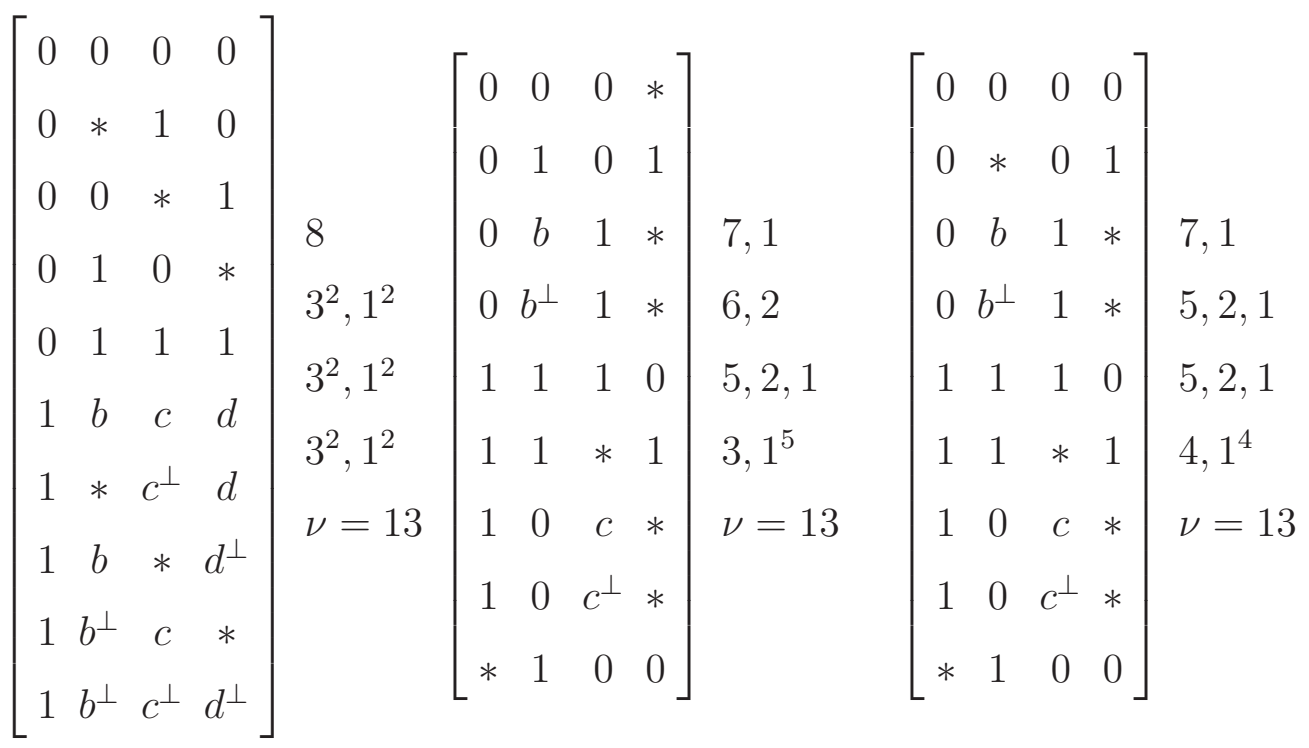

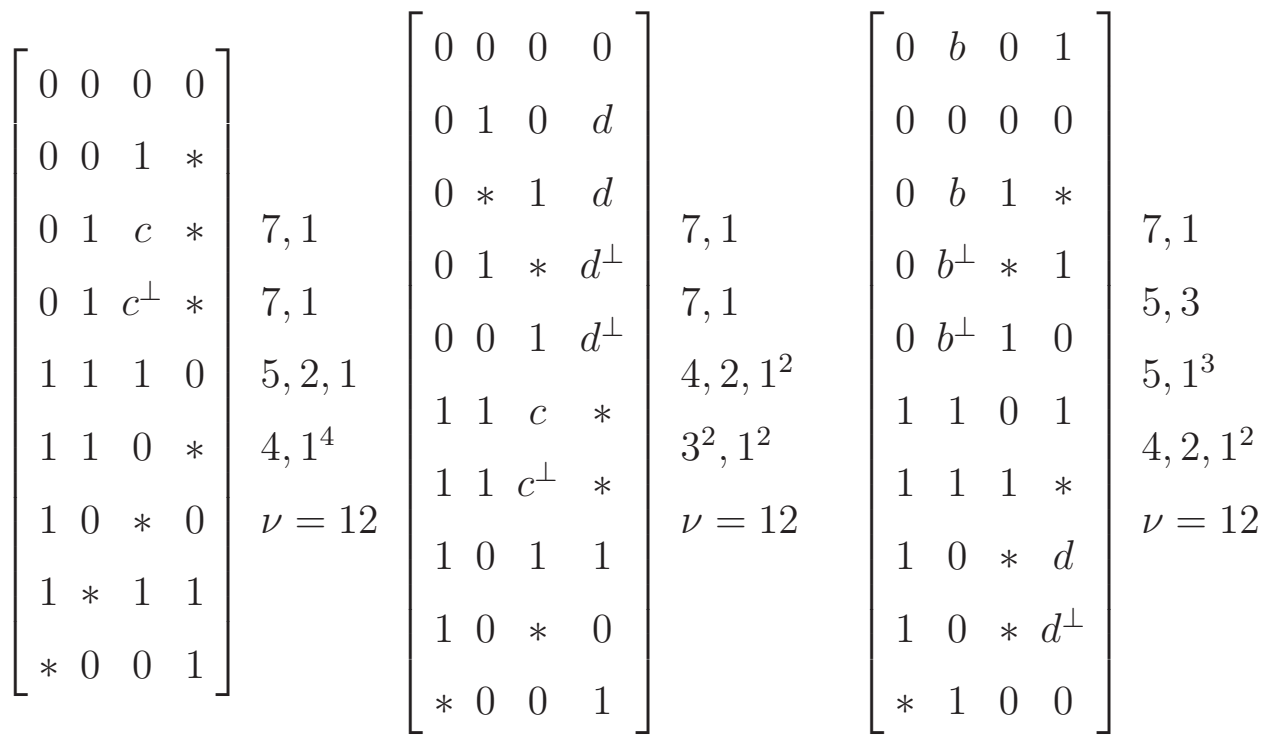




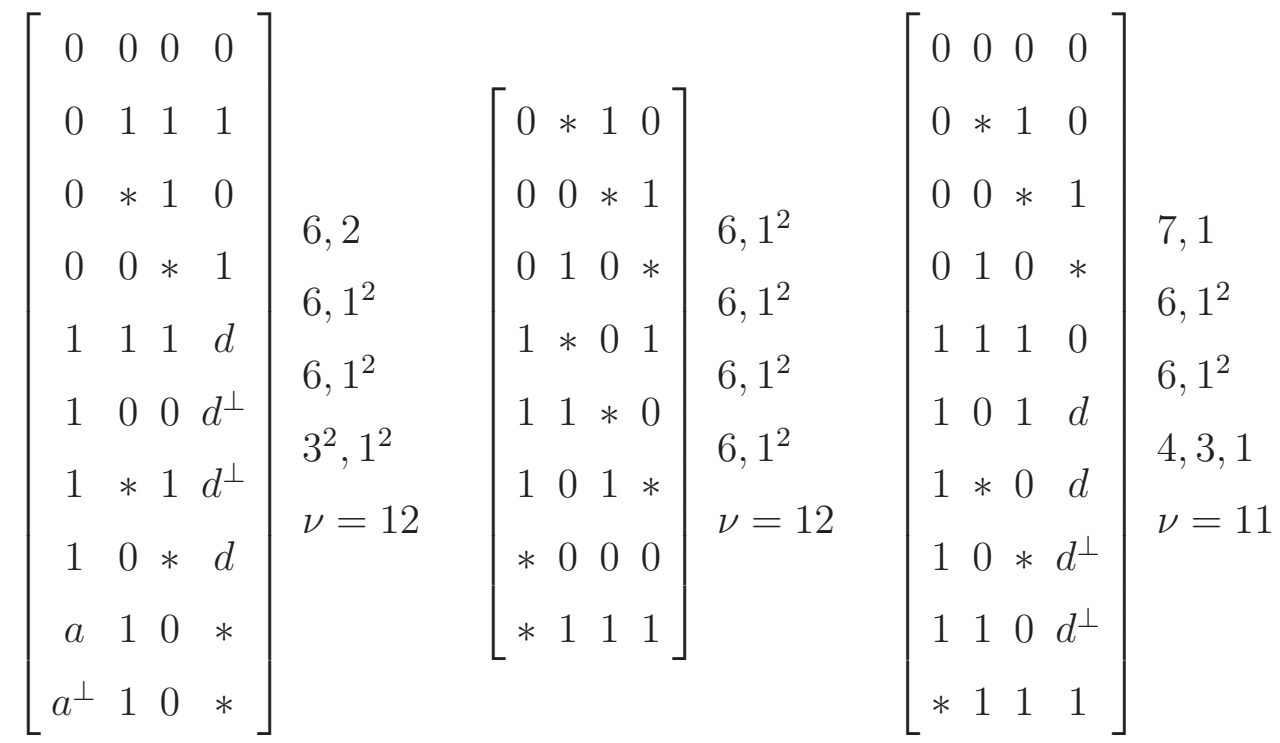

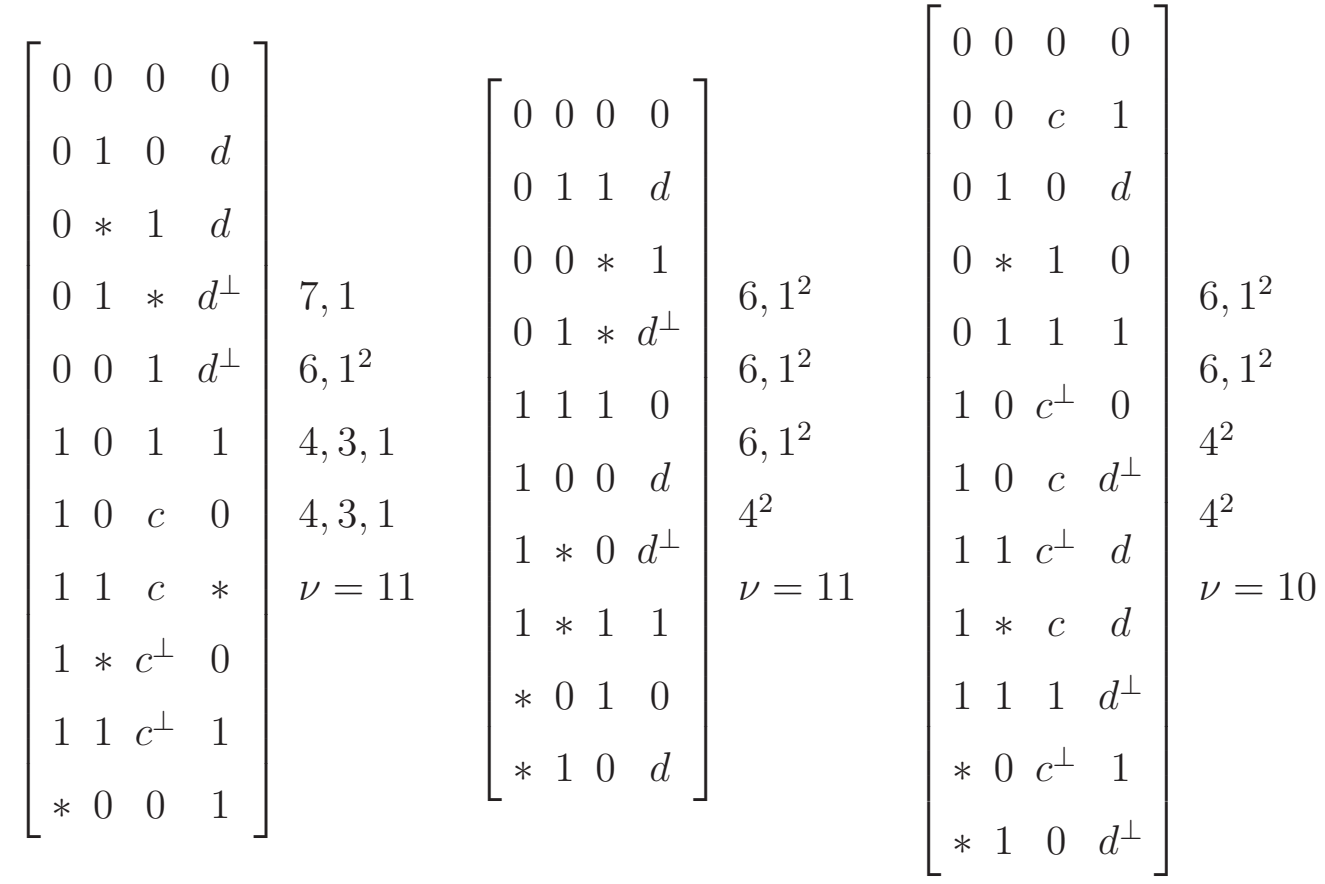

These matrices are arranged so that the parameter $\nu$ decreases from $\nu=15$ to $\nu=10$. For fixed value of $\nu$, the matrices are listed in decreasing lexicographic order of the partitions $\pi_{1}, \pi_{2}, \pi_{3}, \pi_{4}$

Strictly speaking, the above matrices are not members of $\mathcal{O}(4)$ because some columns of these matrices are normalized. We normalize column $j$ if $\mu_{j}>1$ and we choose the normalization so that $\mu(0)=\mu_{j}$. To get the genuine representatives one has to replace in each column the entries 0 and 1 with a new vector variable and its perpendicular, respectively. Of 
course different variables have to be used for different columns. When counting the number of independent variables one has to undo the normalization. We have arranged the columns so that $\pi_{1} \geq \pi_{2} \geq \pi_{3} \geq \pi_{4}$. After each representative $A$ we show the associated partitions $\pi_{i}$ and the parameter $\nu=\nu_{A}$.

For instance let us consider the first matrix in (13). After undoing the normalization, we obtain the matrix

$$
A:=\left[\begin{array}{cccc}
u & v & w & * \\
u & v & w^{\perp} & * \\
u & v^{\perp} & c & * \\
u & v^{\perp} & c^{\perp} & * \\
u^{\perp} & b & x & * \\
u^{\perp} & b & x^{\perp} & * \\
u^{\perp} & b^{\perp} & y & * \\
u^{\perp} & b^{\perp} & y^{\perp} & *
\end{array}\right],
$$

where we used new independent variables $u, v, w$. In this matrix each of the 8 rows really stands for two rows because each entry in the last column is an asterisk. The first column of this matrix contains only one independent variable, say $u$, and its multiplicity is 8 . Consequently, the first partition is $\pi_{1}=8$. The second column has two independent variables, say $v$ and $b$. Each of them has multiplicity 4 , and so $\pi_{2}=4^{2}$. Similarly for the third and fourth columns we obtain the partitions $\pi_{3}=2^{4}$ and $\pi_{4}=1^{8}$. The largest parts of these partitions are $\mu_{1}=8, \mu_{2}=4, \mu_{3}=2$, and $\mu_{4}=1$, respectively. Since $\nu_{i}$ is the number of parts of the partition $\pi_{i}$, we have $\nu_{1}=1, \nu_{2}=2, \nu_{3}=4, \nu_{4}=8$ and so $\nu_{A}=15$.

We number the switching classes in the order that they are listed in (13)-(17). The classes 1,2,4 consist of reducible and the other of irreducible matrices. Each of the switching classes $4,11,12,13,14,15$ consists of just one equivalence class. Each of the other nine switching classes contains at least two equivalence classes. The representatives of these equivalence classes are listed in the appendix. In total there are 33 equivalence classes.

Next we prove our main result, Theorem 19.

Proof. We shall first prove that our list of representatives of the switching classes of maximal matrices of $\mathcal{O}(4)$ is complete. In other words, we have to show that any maximal matrix $A=\left[a_{i, j}\right] \in \mathcal{O}(4)$ is weakly equivalent to one of the matrices listed in (13)-(17).

In this proof, we shall use the notation $\mu_{i}, M_{i}, N_{i}$ introduced just before Lemma 18, Recall 
that each of the submatrices $M_{i}$ of $A$ belongs to $\mathcal{O}(3)$.

Denote by $\pi_{j}$ the partition associated to the column $j$ of $A$. By permuting the columns, we may assume that $\pi_{1} \geq \pi_{2}, \pi_{3}, \pi_{4}$. In spite of this condition, the partition $\pi_{1}$ may vary over a given switching class. Our representatives (as listed in the theorem) are chosen so that $\pi_{1}$ is maximal over all matrices in its switching class.

In view of Lemma 18, we have $\mu_{1} \in\{6,7,8\}$. We divide the proof into four cases according to the partition $\pi_{1}$ associated to the first column of $A$. In each of these four cases we assume that the matrix $A$ is a representative of some switching class (in particular, $A$ is maximal) and that it is chosen so that the partition $\pi_{1}$ is maximal. If during the proof it turns out that $A$ is weakly equivalent to a matrix having bigger partition $\pi_{1}$, then we can discard such A.

Case 1: $\pi_{1}=8$.

Since $A$ is maximal and reducible, both matrices $M_{0}=N_{0}$ and $M_{1}=N_{1}$ must be maximal in $\mathcal{O}(3)$, and moreover no vector variable occurs in $M_{0}$ and $M_{1}$. There are three subcases for the submatrices $M_{0}$ and $M_{1}$ as follows.

1a: they are both reducible;

1b: one of them is reducible and the other irreducible;

1c: they are both irreducible.

Note that the first 8 rows of $A$ as well as the last 8 rows of $A$ are $\{2,3,4\}$-compatible. Hence we can permute arbitrarily the rows of $M_{0}$ as well as those of $M_{1}$. We can also permute arbitrarily and independently the columns of $M_{0}$ and the columns of $M_{1}$ because these operations are switching operations. By using Lemma 14, we infer that each of these three subcases gives a single switching class in $\mathcal{O}(4)$. These are the switching classes $1,2,4$ respectively.

Case 2: $\pi_{1}=7,1$.

By permuting the rows of $A$, we may assume that [ a a a a a a a $\left.b a^{\perp} a^{\perp} a^{\perp} a^{\perp} a^{\perp} a^{\perp} a^{\perp} b^{\perp}\right]^{T}$ is the first column of $A$. Since both $M_{0}, M_{3} \in \mathcal{O}(3)$, Lemma 10 implies that $N_{1}=N_{3}$. Set $x:=a_{8,2}=a_{16,2}, y:=a_{8,3}=a_{16,3}$ and $z:=a_{8,4}=a_{16,4}$.

Subcase 2a: Some $M_{i}$, say $M_{0}$, is reducible.

By permuting the first 7 rows of $A$, we may assume that the first column of $M_{0}$ is $\left[x^{\perp} x^{\perp} x^{\perp} x^{\perp} x x x x\right]^{T}$. By applying Proposition[11(iii) to the submatrix $A[1,2,3,4 ; 3,4] \in$ $\mathcal{O}(2)$, we infer that neither $y$ nor $z$ occurs in it. By using the switching operation of inter- 
changing the two columns of $A[1,2,3,4 ; 3,4]$, we can always assume that $a_{1,3}$ has multiplicity 2 in this submatrix, even after interchanging the last two columns of $A$. By permuting the first four rows of $N_{0}$, we may assume that $a_{1,3}=a_{2,3}=r, a_{3,3}=a_{4,3}=r^{\perp}, a_{1,4}=s$, $a_{2,4}=s^{\perp}, a_{3,4}=t, a_{4,4}=t^{\perp}$, where $r, s, t$ are independent variables which do not occur in $A$ outside the submatrix $A[1,2,3,4 ; 3,4]$.

Suppose that $M_{2}$ is reducible.

Then $y$ or $z$ has multiplicity 4 in $M_{2}$. By interchanging the last two columns of $A$ (if necessary) we may assume that $y$ has multiplicity 4 in $M_{2}$. By permuting the rows of $N_{2}$, we may assume that $a_{i, 3}$ is equal to $y^{\perp}$ for $i=9,10,11,12$ and to $y$ for $i=13,14,15$. By using weak equivalence, we can assume that $a_{9,2}$ has multiplicity 2 in $A[9,10,11,12 ; 2,4] \in$ $\mathcal{O}(2)$. By applying Proposition 11 to this submatrix and by permuting the first four rows of $N_{2}$, we may assume that $a_{9,2}=a_{10,2}=u, a_{11,2}=a_{12,2}=u^{\perp}, a_{9,4}=v, a_{10,4}=v^{\perp}$, $a_{11,4}=w, a_{12,4}=w^{\perp}$, where $u, v, w$ are independent variables which do not occur in $A$ outside the submatrix $A[9,10,11,12 ; 2,4]$. Denote by $p$ and $q$ the multiplicity of $x$ and $y$ in $A[13,14,15,16 ; 2,4] \in \mathcal{O}(2)$ and $A[5,6,7,8 ; 3,4] \in \mathcal{O}(2)$, respectively, and note that $p, q \in\{1,2\}$. If $p=q=1$ then $\pi_{2}=\pi_{3}=5,2,1 ; \pi_{4}=4,1^{4}$ and $A$ belongs to the switching class 6 . Similarly, if $p \neq q$ then $A$ belongs to the switching class 5. If $p=q=2$ then we may assume that $a_{7,3}=y, a_{7,4}=z^{\perp}, a_{15,2}=x$ and $a_{15,4}=z^{\perp}$. By introducing a new vector variable, say $e$, and setting $a_{7,1}=e$ and $a_{15,1}=e^{\perp}$, we obtain a new matrix in $\mathcal{O}(4)$. This contradicts the maximality of $A$. This rules out the possibility $p=q=2$.

Suppose now that $M_{2}$ is irreducible.

If the multiplicity of $x$ in $M_{2}$ is 1 , then we may assume that the first column of $M_{2}$ is [ $u u u u^{\perp} u^{\perp} u^{\perp} x^{\perp} x$ ], and so $\pi_{2}=5,3$. Since $M_{2}$ is equivalent to the matrix (8), we must have $a_{15,3}=y$ and $a_{15,4}=z$. Hence, both $y$ and $z$ have multiplicity 3 in $M_{2}$. By interchanging the two columns of $A[1,2,3,4 ; 3,4]$ if necessary (a switching operation), we may assume that $a_{1,3}$ has multiplicity 2 in that submatrix. If the multiplicity of $y$ in $A[5,6,7,8 ; 3,4] \in \mathcal{O}(2)$ is 1 then $\pi_{3}=4,2,1^{2}$ and $\pi_{4}=5,1^{3}$, and if it is 2 then $\pi_{3}=5,2,1$, $\pi_{4}=4,1^{4}$. In both case $A$ belongs to the switching class 9 .

If the multiplicity of $x$ in $M_{2}$ is 3 , then we may assume that $a_{i, 2}$ is equal to $x^{\perp}$ for $i=11,12,13$ and to $x$ for $i=14,15$. Thus $\pi_{2}=7,1$. Moreover, $u:=a_{9,3}=a_{10,3}$ and $v:=a_{9,4}=a_{10,4}$, and so both $u$ and $v$ have multiplicity 3 in $M_{2}$. As the rows 8 and 9 are orthogonal, we have $u=y^{\perp}$ or $v=z^{\perp}$. Note that it is impossible that both equalities 
hold. By interchanging the last two columns of $A$ (and using the weak equivalence) we may assume that $u=y^{\perp}$ and $v \neq z^{\perp}$.

Suppose that $v=z$. Since $A[9,14,15,16 ; 3,4] \in \mathcal{O}(2)$, we must have $a_{14,4}=a_{15,4}=z^{\perp}$, and we may assume that $a_{11,4}=z^{\perp}$. Consequently, $a_{13,4}=a_{12,4}^{\perp}$. Since $M_{2}$ is irreducible, it follows that $a_{12,3}=a_{13,3}=y$ and $a_{11,3}=y^{\perp}$. As $A[5,6,7,8 ; 3,4] \in \mathcal{O}(2)$, one of $y, z$ must occur twice in this submatrix. If $y$ occurs twice, then we may assume that $a_{5,3}=a_{6,3}=y^{\perp}$, $a_{7,3}=y$ and $a_{7,4}=z^{\perp}$. Moreover, $w:=a_{5,4}=a_{6,4}^{\perp}$ with $w$ and $z$ independent. By interchanging the two columns of the submatrix $A[1,2,3,4 ; 3,4] \in \mathcal{O}(2)$ if necessary (a switching operation), we may assume that $a_{1,3}$ has multiplicity 2 in this submatrix. Hence, $\pi_{3}=5,2,1, \pi_{4}=4,1^{4}$ and $A$ belongs to the switching class 7 . Similarly, if $z$ occurs twice in $A[5,6,7,8 ; 3,4]$ we can verify that $A$ again belongs to the switching class 7 .

Finally suppose that $v$ and $z$ are independent, and so $z$ occurs only once in $M_{2}$. As $P:=A[10,11,12,13 ; 3,4] \in \mathcal{O}(2)$ and $z$ does not occur in $P$, we may assume that $a_{11,4}=v$, $a_{i, 4}=v^{\perp}$ for $i=12,13,14$ and $a_{15,4}=z^{\perp}$. It follows now that $a_{11,3}=a_{15,3}=y$ and $a_{14,3}=y^{\perp}$. If $y$ has multiplicity 2 in $Q:=A[5,6,7,8 ; 3,4] \in \mathcal{O}(2)$, then we may assume that $a_{7,3}=y$ and $a_{7,4}=z^{\perp}$. But then we can set $a_{7,1}=w$ and $a_{15,1}=w^{\perp}$, where $w$ is a new vector variable, to obtain a new matrix in $\mathcal{O}(4)$. This contradicts the maximality of $A$. We conclude that $y$ has multiplicity 1 in $Q$ and $z$ multiplicity 2 . Hence $\pi_{3}=4,2,1^{2}, \pi_{4}=3^{2}, 1^{2}$ and $A$ belongs to the switching class 8 .

Subcase 2b: All $M_{i}$ are irreducible.

Let us introduce two submatrices $P:=A[2,3,4,5 ; 3,4]$ and $Q:=A[10,11,12,13 ; 3,4]$. Note that each row of the matrix (8) contains at most one entry of multiplicity 1 . As $M_{0}$ is irreducible, it is equivalent to the matrix (8) and so at least two of the entries $x, y, z$ must have multiplicity 3 in $M_{0}$. We may assume that this holds true for $x$ and $y$. For the same reason, at least one of $x, y$, say $x$, has multiplicity 3 in $M_{2}$ and so $\pi_{2}=6,1^{2}$. We may assume that $a_{i, 2}$ is equal to $x^{\perp}$ for $i=3,4,5,11,12,13$ and equal to $x$ for $i=6,7,14,15$. Consequently, $a_{1,3}=a_{2,3}, a_{1,4}=a_{2,4}$ and $v:=a_{9,3}=a_{10,3}, w:=a_{9,4}=a_{10,4}$. We infer that $a_{1,3}$ and $a_{1,4}$ have multiplicity 3 in $M_{0}$, and $v$ and $w$ have multiplicity 3 in $M_{2}$. Since both $a_{1,3}$ and $y$ have multiplicity 3 in $M_{0}$, we must have $a_{1,3} \in\left\{y, y^{\perp}\right\}$. Note that the submatrices $P$ and $Q$ belong to $\mathcal{O}(2)$.

Suppose that $a_{1,3}=y$.

Since rows 1 and 8 are orthogonal, we have $a_{1,4}=a_{2,4}=z^{\perp}$. By permuting the rows 
$3,4,5$ (if necessary), we may assume that $a_{3,3}=y^{\perp}$. It follows that $a_{3,4}=z^{\perp}$. Thus $z^{\perp}$ has multiplicity 2 in the submatrix $P$. Therefore we have $a_{4,4}=a_{5,4}=z$. Now observe that we can apply Proposition 11 (iii) to the submatrix $A[4,5 ; 3] \in \mathcal{O}(1)$. We infer that the variable $a_{4,3}=a_{5,3}^{\perp}$ has multiplicity 1 in $A$. As we must have $a_{7,3}=a_{8,3}=y^{\perp}$, the same argument shows that the variable $a_{6,4}=a_{7,4}^{\perp}$ has multiplicity 1 in $A$. Since row 8 and 9 are orthogonal, $v=y^{\perp}$ or $w=z^{\perp}$. Note that we cannot have $v=y^{\perp}$ and $w=z^{\perp}$.

We claim that $w \neq z$ and $v \neq y$. If $w=z$ then $z$ has multiplicity 3 in $M_{2}$ and we may assume that $a_{11,4}=z^{\perp}$. By Corollary [17, applied to the rows 3 and 8 of $M_{2}$, we have $a_{11,3}=y^{\perp}$. By applying Proposition 11 (iii) to the submatrix $A[3,11 ; 1] \in \mathcal{O}(1)$, we obtain a contradiction. We conclude that $w \neq z$. Similarly, $v \neq y$. Thus our claim is proved.

Assume that $v=y^{\perp}$. If $y$ occurs twice in the submatrix $Q$, then we may assume that $a_{11,3}=y^{\perp}$. By inspecting the matrix (8), we infer that $a_{11,4}=z^{\perp}$. By applying Proposition 11 (iii) to the submatrix $A[3,11 ; 1] \in \mathcal{O}(1)$, we obtain a contradiction. We conclude that $y$ occurs only once in $Q$ and we may assume that $a_{11,3}=y$ and $a_{13,3}^{\perp}=a_{14,3}=y$. It follows easily that $a_{15,4}=z^{\perp}, a_{14,4}=w^{\perp}, a_{12,4}=a_{13,4}=w^{\perp}$ and $a_{11,3}=w$. We have $\pi_{3}=6,1^{2}$ and $\pi_{4}=4,3,1$. This $A$ belongs to the switching class 12 .

Assume now that $v$ and $y$ are independent. Then we must have $w=z^{\perp}$. Since $y$ occurs only once in $M_{2}$, we may assume that $a_{15,3}=y^{\perp}$. It follows that $v$ has multiplicity 2 in $Q$, and we may assume that $a_{11,3}=v$ and $a_{12,3}=a_{13,3}=a_{14,3}=v^{\perp}$. It follows easily that $a_{11,4}=z$ and $a_{15,4}=a_{14,4}^{\perp}=z$. Hence, $\pi_{3}=4,3,1$ and $\pi_{4}=6,1^{2}$. This $A$ belongs also to the switching class 12 .

Suppose that $a_{1,3}=y^{\perp}$.

For convenience set $u:=a_{1,4}$. Since $a_{2,3}=a_{1,3}=y^{\perp}$ and $P \in \mathcal{O}(2)$, by permuting the rows $3,4,5$ of $A$, we can assume that $a_{3,3}=y$. The variable $y$ may occur in $P$ once or twice. We distinguish these two possibilities.

Assume that $y$ occurs only once in $P$. Since $y$ has multiplicity 3 in $M_{0}$, we may assume that $a_{7,3}=a_{6,3}^{\perp}=y$. Since the rows 1 and 6 are orthogonal, we have $a_{6,4}=u^{\perp}$. Since the rows 7 and 8 are orthogonal, we have $a_{7,4}=z^{\perp}$. Moreover, Proposition 11 (iii) applied to $M_{0}$ and its submatrix $A[7,8 ; 4]$ implies that $z$ occurs only once in $M_{0}$. Thus, $u$ and $z$ are independent. Note that $a_{4,3}$ occurs only once in $M_{0}$ and that $a_{5,3}=a_{4,3}^{\perp}$. This implies that $a_{4,4}=a_{5,4}$. As $u$ has multiplicity 3 in $M_{0}$, it follows that $a_{3,4}=u$ and $a_{4,4}=a_{5,4}=u^{\perp}$. As $A$ is maximal, by Proposition 11 (iii), we have $\mu\left(a_{4,3}\right)=1$. 
There are three possibilities for the variable $v$ as follows: $v=y, v=y^{\perp}$ and $v, y$ are independent.

First, let $v=y$. Since rows 8 and 9 are orthogonal, we have $w=z^{\perp}$, i.e., $a_{9,4}=a_{10,4}=z^{\perp}$. Since $y$ has multiplicity 3 in $M_{2}$, we must have $a_{14,3}=a_{15,3}=y^{\perp}$ and $a_{14,4}=a_{15,4}^{\perp}$. By permuting the rows $11,12,13$ of $A$, we may assume that $a_{11,3}=y^{\perp}$. Hence, the entry $a_{12,3}$ occurs only once in $M_{2}$ and $a_{13,3}=a_{12,3}^{\perp}$. This implies that $a_{12,4}=a_{13,4}$. As $A$ is maximal, by Proposition 11 (iii), we have $\mu\left(a_{12,3}\right)=\mu\left(a_{14,4}\right)=1$. Now one can verify that $A$ belongs to the switching class 12 .

Second, let $v=y^{\perp}$. At least one of the entries $a_{14,3}, a_{15,3}$ is not equal to $y^{\perp}$. By interchanging the rows 14 and 15 if necessary, we may assume that $a_{15,3} \neq y^{\perp}$. As rows 15 and 16 are orthogonal, we must have $a_{15,4}=z^{\perp}$. We cannot have $a_{15,3}=y$ since then by Proposition 11 (iii) applied to $A$ and its submatrix $A[7,15 ; 1]$ would give a contradiction. We infer that $y$ must occur twice in $Q$ and that $\mu\left(a_{14,3}\right)=1$. By permuting the rows $11,12,13$ we may assume that $a_{12,3}=y^{\perp}$ and $a_{13,3}=a_{14,3}=y$. By Proposition 11 (iii) applied to $A$ and its submatrix $A[12,13 ; 4]$, we deduce that $\mu\left(a_{12,4}\right)=1$. Since rows 14 and 16 are orthogonal, we must have $w=z$. Now one can verify that $A$ belongs to the switching class 12 .

Third, let $v$ and $y$ be independent. Then $v$ has multiplicity 2 in $Q$ and we may assume that $a_{11,3}=v, a_{12,3}=a_{13,3}=a_{14,3}=v^{\perp}$ and $a_{15,3}=y^{\perp}$. Since rows 9 and 16 are orthogonal, we must have $w=z^{\perp}$. Now one can verify that $A$ belongs to the switching class 13 .

Now assume that $y$ occurs twice in $P$. By permuting the rows 3,4,5 of $A$ we may assume that $a_{3,3}=y^{\perp}$ and $a_{4,3}=a_{5,3}=y$. By inspecting the matrix (8), we infer that we must have $a_{3,4}=z^{\perp}$. Since the rows 2 and 3 are orthogonal, we must have $u=z$. By applying Proposition 11 (iii) to $A$ and the submatrix $A[4,5 ; 4]$, we conclude that $a_{4,4}=a_{5,4}^{\perp}$ and $\mu\left(a_{4,4}\right)=1$. Since $z=u$ has multiplicity 3 in $M_{0}$, we must have $a_{6,4}=a_{7,4}=z^{\perp}$.

Since the rows 8 and 9 are orthogonal, $v=y^{\perp}$ or $w=z^{\perp}$. Note that we cannot have $v=y^{\perp}$ and $w=z^{\perp}$. If $w=z$ then $z$ has multiplicity 3 in $M_{2}$ and we may assume that $a_{11,4}=z^{\perp}$. By applying Corollary 17 to the rows 3 and 8 of $M_{2}$, we obtain that $a_{11,3}=y^{\perp}$. By applying Proposition 11 (iii) to the submatrix $A[3,11 ; 1]$, we obtain a contradiction. We conclude that $w \neq z$. Similarly, $v \neq y$.

Thus, if $v=y^{\perp}$ then $w$ and $z$ are independent, and $z$ must occur only once in $M_{2}$. It follows that $z^{\perp}$ does not occur in $Q$. Hence, we may assume that $a_{11,4}=w, a_{12,4}=$ $a_{13,4}=a_{14,4}=w^{\perp}$ and $a_{15,4}=z^{\perp}$. By applying Proposition 11](iii) to $A$ and the submatrix 
$A[12,13 ; 3]$, we obtain that $a_{12,3}=a_{13,3}^{\perp}$ and $\mu\left(a_{13,3}\right)=1$. It follows that $a_{14,3}=y^{\perp}$ and $a_{15,4}=y$. Thus, $\pi_{3}=6,1^{2}, \pi_{4}=4,3,1$ and one can verify that $A$ belongs to the switching class 12. Similarly, if $w=z^{\perp}$ then $v$ and $y$ are independent, $\pi_{3}=4,3,1, \pi_{4}=6,1^{2}$ and $A$ belongs to the switching class 12 .

Case 3: $\pi_{1}=6,2$.

By permuting the rows of $A$ and renaming the variables, if necessary, we may assume that $\left[\text { a } a \text { a } a \text { a } a \text { a } b \quad b \quad a^{\perp} a^{\perp} a^{\perp} a^{\perp} a^{\perp} a^{\perp} b^{\perp} b^{\perp}\right]^{T}$ is the first column of $A$. Assume that $a_{7, j} \neq a_{8, j}$ for at least two indices $j \in\{2,3,4\}$. Since both submatrices

$$
M_{0}=\left[\begin{array}{l}
N_{0} \\
N_{1}
\end{array}\right], \quad M_{3}=\left[\begin{array}{l}
N_{3} \\
N_{0}
\end{array}\right]
$$

belong to $\mathcal{O}(3)$, Lemma 16 implies that the $2 \times 3$ submatrices $N_{1}$ and $N_{3}$ may differ only in the order of rows. By interchanging the last two rows of $A$ (if necessary) we may assume that $N_{1}=N_{3}$. This switch will not change the first column of $A$ because $a_{15,1}=a_{16,1}=b^{\perp}$. It is now easy to verify that if we replace the entries $a_{8,1}=b$ and $a_{16,1}=b^{\perp}$ in $A$ with new vector variables $x$ and $x^{\perp}$, respectively, then the modified matrix $A$ will still belong to $\mathcal{O}(4)$. This gives a contradiction since $A$ is maximal. Hence, the equality $a_{7, j}=a_{8, j}$ must hold for exactly two indices $j \in\{2,3,4\}$. We may assume that $c:=a_{7,2}=a_{8,2}$ and $d:=a_{7,3}=a_{8,3}$.

Since $M_{0}, M_{3} \in \mathcal{O}(3)$, we must also have $a_{15,2}=a_{16,2}=c$ and $a_{15,3}=a_{16,3}=d$. Since the rows 7 and 8 of $A$ are orthogonal, we must have $a_{8,4}=a_{7,4}^{\perp}$. Similarly, $a_{16,4}=a_{15,4}^{\perp}$. Since $A$ is maximal, by applying Proposition 11 (iii) to the submatrix $A[7,8 ; 4]$, we deduce that $\mu\left(a_{7,4}\right)=1$. Similarly, $\mu\left(a_{15,4}\right)=1$. In particular, the vector variables $a_{7,4}$ and $a_{15,4}$ are independent.

Subcase 3a: Some $M_{i}$, say $M_{0}$, is reducible.

Since $\mu\left(a_{7,4}\right)=1$, either $c$ or $d$, say $c$, must have multiplicity 4 in $M_{0}$. Thus there are four entries $c$ in the first column of $M_{0}$ and at least two entries $c$ in the first column of $M_{2}$. As $\mu(x) \leq 6$ for all entries $x$ of $A$, we conclude that $\mu(c)=6$. Hence, $c$ has multiplicity 2 in $M_{2}$. By Corollary 17, $M_{2}$ is reducible. By permuting the first six rows of $A$, we may assume that the first column of $M_{0}$ is $\left[c^{\perp} c^{\perp} c^{\perp} c^{\perp} c c c c c\right]^{T}$. By permuting the rows 9 to 14 of $A$, we may also assume that $a_{9,2}=a_{10,2}=c^{\perp}$. As $\mu\left(c^{\perp}\right)=6$, we have $a_{i, 2} \neq c^{\perp}$ for $i \in\{11,12,13,14\}$. Since the row 15 of $A$ is orthogonal to the rows $5,6,11,12,13,14$ and $\mu\left(a_{15,4}\right)=1$, we deduce that $a_{i, 3}=d^{\perp}$ for $i \in\{5,6,11,12,13,14\}$. It follows that 
$a_{5,4}=a_{6,4}^{\perp}$ and that the multiplicity of $d$ in $M_{2}$ must be 4 . Consequently, $a_{9,3}=a_{10,3}=d$, and $a_{9,4}=a_{10,4}^{\perp}$. By applying Proposition 11 (iii) to the submatrices $A[5,6 ; 4]$ and $A[9,10 ; 4]$, we deduce that $\mu\left(a_{5,4}\right)=\mu\left(a_{9,4}\right)=1$.

Note that the submatrices $A[1,2,3,4 ; 3,4]$ and $A[11,12,13,14 ; 2,4]$ must belong to $\mathcal{O}(2)$. By using Proposition 11 (iii-iv) we deduce that these two submatrices are maximal in $\mathcal{O}(2)$ and have no vector variable in common. Hence, these submatrices are equivalent to the second matrix in (5). By applying the switching operations (if necessary) on these two submatrices, we may assume that $a_{1,3}$ and $a_{11,2}$ have multiplicity 2 in $A[1,2,3,4 ; 3,4]$ and $A[11,12,13,14 ; 2,4]$, respectively. Then we have $\pi_{1}=\pi_{2}=\pi_{3}=6,2$ and $\pi_{4}=1^{8}$. Thus $A$ is weakly equivalent to the third matrix in (13), i.e., $A$ belongs to the switching class 3 .

Subcase 3b: All $M_{i}$ are irreducible.

Thus, each $M_{i}$ is equivalent to the matrix (8). By Corollary 17, both $c$ and $d$ must have multiplicity 3 in both $M_{0}$ and $M_{1}$. Hence, $\mu(c)=6$. By permuting the rows of $N_{0}$ and $N_{2}$, we may assume that $\left[x x^{\perp} c^{\perp} c^{\perp} c^{\perp} c c c y y^{\perp} c^{\perp} c^{\perp} c^{\perp} c c c\right]^{T}$ is the second column of $A$. As $M_{0}$ and $M_{2}$ are irreducible and $c$ is independent from $x$ and $y$, by inspecting the matrix (8), we deduce that $a_{1,3}=a_{2,3}, u:=a_{1,4}=a_{2,4}, a_{9,3}=a_{10,3}$, and $v:=a_{9,4}=a_{10,4}$. By Corollary 17. $u$ and $v$ have multiplicity 3 in $M_{0}$ and $M_{2}$, respectively. As each of the rows $1,2,6,9,10,14$ of $A$ is orthogonal to rows 7 and 8 , we infer that $a_{i, 3}=d^{\perp}$ for $i=1,2,6,9,10,14$. As rows 1 and 6 of $A$ are orthogonal, we have $a_{6,4}=u^{\perp}$. As rows 9 and 14 of $A$ are orthogonal, we have $a_{14,4}=v^{\perp}$.

Exactly one of the entries $a_{i, 3}, i=3,4,5$ is equal to $d$. By permuting the rows $3,4,5$ of $A$, we may assume that $a_{5,3}=d$. Similarly, we may assume that $a_{13,3}=d$.

Since $a_{5,2}=a_{6,2}^{\perp}$ and $a_{5,3}=a_{6,3}^{\perp}$, by inspection of (8) , we infer that we must also have $a_{5,4}=a_{6,4}^{\perp}$, i.e., $a_{5,4}=u$. Similarly, $a_{13,4}=v$. It follows that $a_{3,4}=a_{4,4}=u^{\perp}$ and $a_{11,4}=a_{12,4}=v^{\perp}$.

By applying Proposition 11 (iii) to the $2 \times 1$ submatrix $A[1,2 ; 2]$, we deduce that $x$ and $y$ must be independent. Similarly, $a_{3,3}$ and $a_{11,3}$ must be independent, as well as $a_{7,4}$ and $a_{15,4}$. Finally, the maximality of $A$ implies that $u$ and $v$ are independent. Thus $\pi_{2}=\pi_{3}=6,1^{2}$ and $\pi_{4}=3^{2}, 1^{2}$, and $A$ belongs to the switching class 10 .

Case 4: $\pi_{1}=6,1^{2}$.

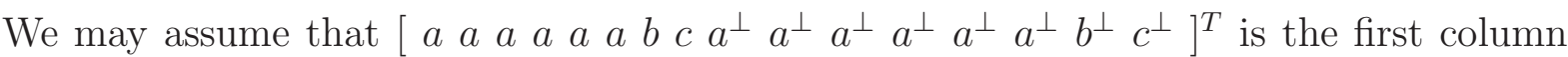
of $A$. Since both $M_{1}, M_{2} \in \mathcal{O}(3)$, Lemma 10 implies that $N_{1}=N_{4}$. Similarly, since 
$M_{2}, M_{3} \in \mathcal{O}(3)$, we have $N_{2}=N_{5}$. Set $x:=a_{7,2}, y:=a_{7,3}$ and $z:=a_{7,4}$. As $N_{1}=N_{4}$ we also have $a_{15,2}=x, a_{15,3}=y$ and $a_{15,4}=z$. As $N_{2}=N_{5}$, we have $a_{16, j}=a_{8, j}$ for $j=2,3,4$.

Subcase 4a: Some $M_{i}$, say $M_{0}$, is reducible.

Since $M_{0}$ is reducible, at least one of $x, y, z$ must have multiplicity 4 in $M_{0}$. By permuting the last three columns of $A$ (if necessary) we may assume that $x$ has multiplicity 4 in $M_{0}$. In particular, $a_{8,2} \in\left\{x, x^{\perp}\right\}$.

Suppose that $a_{8,2}=x^{\perp}$. Then also $a_{16,2}=x^{\perp}$.

Assume that $M_{3}$ is irreducible. Then Corollary 17 and $\mu(x) \leq 6$ imply that $x$ must occur only once in $M_{3}$. Since $M_{3}$ is equivalent to the matrix (8), we conclude that $a_{16,3}=y$ and $a_{16,4}=z$. It follows that also $a_{8,3}=y$ and $a_{8,4}=z$. We can now select a new independent variable $r$ and replace the entries $x$ and $x^{\prime}$ with $r$ and $r^{\perp}$ respectively, but only in the four positions $a_{i, 2}, i=7,8,15,16$. We obtain a new matrix in $\mathcal{O}(4)$ showing that $A$ is not maximal. This contradicts our hypothesis. We conclude that $M_{3}$ must be reducible.

By permuting the first 6 rows of $A$, we may assume that $a_{i, 2}$ is equal to $x^{\perp}$ for $i=1,2,3$ and equal to $x$ for $i=4,5,6$. As $\mu(x) \leq 6$, the multiplicity of $x$ in $M_{3}$ is 1 or 2 . Hence, either $y$ or $z$ must have multiplicity 4 in $M_{3}$. By interchanging the last two columns of $A$ (if necessary) we may assume that $y$ has multiplicity 4 in $M_{3}$. As $a_{8,3}=a_{16,3}$, we have $a_{8,3} \in\left\{y, y^{\perp}\right\}$. By permuting the rows of $N_{3}$, we may assume that $a_{i, 3}$ is equal to $y^{\perp}$ for $i=9,10,11$, it is equal to $y$ for $i=13,14$, and that $a_{12,3}=a_{8,3}^{\perp}$.

If $a_{8,3}=y$ then either $x$ or $z$ must have multiplicity 2 in the submatrix $A[13,14,15,16 ; 2,4] \in$ $\mathcal{O}(2)$. If $z$ has multiplicity 2 , then $z=u^{\perp}$ and $\mu(z)=6$. We can interchange the two columns of $A[9,10,11,12 ; 2,4] \in \mathcal{O}(2)$ (a switching operation) to obtain a matrix in $\mathcal{O}(4)$ having $\pi_{4}=6,2$. This contradicts our hypothesis that $A$ is chosen in its switching class to have the largest possible partition $\pi_{1}$. A similar argument gives a contradiction if $x$ has multiplicity 2 in $A[13,14,15,16 ; 2,4]$.

Thus, we may assume that $a_{8,3}=y^{\perp}$, and consequently $a_{16,3}=y^{\perp}$ and $a_{12,3}=y$. Since the submatrix $A[12,13,14,15 ; 2,4] \in \mathcal{O}(2)$ and $a_{15,2}=x$, the variable $x^{\perp}$ must also occur in this submatrix. By permuting the rows $12,13,14$ of $A$, we may assume that $a_{14,2]=x^{\perp}}$. We infer that $\mu\left(x^{\perp}\right)=6$ and that $r:=a_{12,2}=a_{13,2}^{\perp}$, where the variable $r$ is independent from $x$. Since $x$ has multiplicity 1 in $A[12,13,14,15 ; 2,4]$, the entry $a_{15,4}=z$ must have multiplicity 2 in this submatrix. It follows that $a_{14,4}=z$ and $a_{12,4}=a_{13,4}=z^{\perp}$. Since $\mu(x)=\mu\left(x^{\perp}=6\right.$, by permuting the rows $9,10,11$ of $A$, we may assume that $a_{9,2}=x$. It 
follows that $s:=a_{10,2}=a_{11,2}^{\perp}$ where $s$ is independent from $x$. Since $x$ has multiplicity 1 in $A[8,9,10,11 ; 2,4] \in \mathcal{O}(2)$, the entry $a_{8,4}=u^{\perp}$ must have multiplicity 2 in this submatrix. It follows that $a_{9,4}=u^{\perp}$ and $a_{10,4}=a_{11,4}=u$. As $\mu(z) \leq 6, u$ and $z$ must be independent. Since $A$ is maximal, $r$ and $s$ must be also independent. Hence $A$ belongs to the switching class 14 .

Suppose now that $a_{8,2}=x$. Then also $a_{16,2}=x$.

We may assume that $a_{i, 2}$ is equal to $x$ for $i=5,6$ and it is equal to $x^{\perp}$ for $i=1,2,3,4,9,10$. Since $\pi_{1} \geq \pi_{2}$, by permuting the last four rows of $N_{3}$, we may assume that $a_{11,2}=u$, $a_{12,2}=u^{\perp}, a_{13,2}=v, a_{14,2}=v^{\perp}$, with $u, v, x$ independent.

If $a_{8,3}=y$ then also $a_{16,3}=y$ and $a_{8,4}=a_{16,4}=z^{\perp}$. It follows easily that $a_{i, 3}=y^{\perp}$ for $i=11,12,13,14$. By interchanging the two columns of $A[11,12,13,14 ; 2,4]$ (a switching operation) we see that $A$ is weakly equivalent to a matrix with $\pi_{2}=6,2$. As $\pi_{2}>\pi_{1}=6,1^{2}$, we can discard this $A$. Similarly, we can discard $A$ if $a_{8,4}=z$.

Since the rows 7 and 8 of $A$ are orthogonal, by interchanging the last two columns of $A$ (if necessary) we may assume that $a_{8,4}=a_{16,4}=z^{\perp}$.

For convenience set $w:=a_{8,3}$ and recall that $w \neq y$. Note that the submatrix $A[12,14,15,16 ; 3,4] \in \mathcal{O}(2)$. Assume that at least one of $a_{12,4}$ and $a_{14,4}$ is independent from $z$, say $a_{12,4}$. Then $a_{12,3}$ has to be orthogonal to both $w$ and $y$. This is impossible because $w \neq y$. Thus, $z$ must have multiplicity 2 in $A[12,14,15,16 ; 3,4]$. By permuting the rows of $N_{3}$ (if necessary), we may assume that $a_{12,4}=a_{14,4}^{\perp}=z$. Since the rows 12 and 15 of $A$ are orthogonal, we infer that $a_{12,3}=y^{\perp}$. Since the rows 14 and 16 of $A$ are orthogonal, we infer that $a_{14,3}=w^{\perp}$. Since also $A[12,13,15,16 ; 3,4] \in \mathcal{O}(2)$, Lemma 10 implies that $a_{13,3}=a_{14,3}=w^{\perp}$ and $a_{13,4}=a_{14,4}=z^{\perp}$. Similarly, since $A[11,14,15,16 ; 3,4] \in \mathcal{O}(2)$ we have $a_{11,3}=a_{12,3}=y^{\perp}$ and $a_{11,4}=a_{12,4}=z$. Assume that $a_{9,4}$ and $z$ are independent. Then, since $A[9,10,11,13 ; 3,4] \in \mathcal{O}(2)$, the entry $a_{9,3}$ must be orthogonal to both $a_{11,3}=y^{\perp}$ and $a_{13,3}=w^{\perp}$. As $w \neq y$, this is impossible. Thus, we must have $\left\{a_{9,4}, a_{10,4}\right\}=\left\{z, z^{\perp}\right\}$. By interchanging the rows 9 and 10 of $A$ (if necessary), we may assume that $a_{9,4}=z$ and $a_{10,4}=z^{\perp}$. Since rows 9 and 11 are orthogonal, we must have $a_{9,3}=y$. Since rows 10 and 13 are orthogonal, we must have $a_{10,3}=w$. Assume that $z$ occurs twice in $A[5,6,7,8 ; 3,4] \in \mathcal{O}(2)$. Then $\mu(z)=6$ and by interchanging the two columns of $A[1,2,3,4 ; 3,4] \in \mathcal{O}(2)$ (a switching operation), the last column of $A$ will have 6,2 as the associated partition. This contradicts our choice of $A$. We conclude that $z$ must 
occur only once in $A[5,6,7,8 ; 3,4]$. By interchanging the rows 5 and 6 of $A$ (if necessary), we may assume that $a_{5,4}=a_{6,4}^{\perp}=r$, where $r$ is a variable independent from $z$. Since the row 6 of $A$ is orthogonal to rows 7 and 8 , we infer that $a_{5,3}$ is orthogonal to both $w$ and $y$. As $w \neq y$ this is impossible.

Subcase 4b: All $M_{i}$ are irreducible.

Since the rows 7 and 8 are orthogonal, by permuting the last three columns of $A$, we may assume that $a_{8,2}=x^{\perp}$. We discuss three cases, namely $a_{8,3} \in\left\{y, y^{\perp}\right\}$ and $a_{8,3}$ and $y$ are independent.

Suppose that $a_{8,3}=y^{\perp}$. As $M_{0}$ is irreducible, we must have also $a_{8,4}=z^{\perp}$. Since $N_{5}=N_{2}$ and $M_{0}$ and $M_{3}$ are equivalent to (8), we infer that each of the variables $x, y, z$ has multiplicity 3 in $M_{0}$ and in $M_{3}$. Hence, by permuting the rows of $N_{0}$ and those of $N_{3}$, we may assume that $u:=a_{1,2}=a_{2,2}^{\perp}, v:=a_{3,3}=a_{4,3}^{\perp}, w:=a_{5,4}=a_{6,4}^{\perp}$ and $r:=a_{9,2}=a_{10,2}^{\perp}, s:=a_{11,3}=a_{12,3}^{\perp}$, $t:=a_{13,4}=a_{14,4}^{\perp}$. Since $A$ is maximal, the vector variables $u, v, w, r, s, t, x, y, z$ must be independent. By interchanging the rows 7 and 8 as well as the rows 15 and 16, we may assume that $a_{3,2}=a_{4,2}=x$ and $a_{5,2}=a_{6,2}=x^{\perp}$. Then it follows that $a_{1,3}=a_{2,3}=y^{\perp}$, $a_{5,3}=a_{6,3}=y, a_{1,4}=a_{2,4}=z, a_{3,4}=a_{4,4}=z^{\perp}$. There are now two possibilities for the block $N_{3}$. First, $a_{i+8, j}=a_{i, j}$ for $j=2,3,4$ if $a_{i, j}$ is independent from $u, v, w$. In that case $A$ is not maximal as we can replace the entries $a_{1,1}$ and $a_{2,1}$ with $p$ and the entries $a_{9,1}$ and $a_{10,1}$ with $p^{\perp}$, where $p$ is a new independent variable. The new matrix is still in $\mathcal{O}(4)$, which contradicts the maximality of $A$. Thus we can discard this possibility. Second, $a_{i+8, j}=a_{i, j}^{\perp}$ for $j=2,3,4$ if $a_{i, j}$ is independent from $u, v, w$. In that case $A$ is equivalent to the representative of the switching class 11, i.e., the second matrix in (15).

Suppose that $a_{8,3}=y$. As $y$ has multiplicity 3 in $M_{0}$ and $M_{3}$, we may assume that the third column of $A$ is [ $u u^{\perp} y^{\perp} y^{\perp} y^{\perp}$ y y y $v v^{\perp} y^{\perp} y^{\perp} y^{\perp}$ y y y $]^{T}$ where $u, v, y$ are independent. Since $M_{0}$ and $M_{3}$ are irreducible, we must have $a_{1,2}=a_{2,2}, a_{1,4}=a_{2,4}$ $a_{10,2}=a_{9,2}$ and $a_{10,4}=a_{9,4}$.

Assume that the multiplicity of $x$ in $M_{0}$ is 1 . Then we must have $a_{8,4}=z, a_{16,4}=z$, and so $x$ has also multiplicity 1 in $M_{3}$. By interchanging the two columns of the submatrix $A[7,8,15,16 ; 1,2] \in \mathcal{O}(2)$ (a switching operation), we obtain a matrix in $\mathcal{O}(4)$ with 6,2 as the partition associated to the first column. Hence, we can discard this possibility.

We conclude that the multiplicity of $x$ in $M_{0}$ and in $M_{3}$ is 3 . By inspection of the matrix (8), we conclude that $p:=a_{8,4}$ and $z$ are independent. Since rows 1 and 6 are orthogonal to 
rows 7 and 8, we must have either

$$
a_{1,2}=x, a_{1,4}=z^{\perp}, a_{6,2}=x^{\perp}, a_{6,4}=p^{\perp}
$$

or

$$
a_{1,2}=x^{\perp}, a_{1,4}=p^{\perp}, a_{6,2}=x, a_{6,4}=z^{\perp} .
$$

The same two alternatives apply to the corresponding entries of $M_{3}$, namely either

$$
a_{9,2}=x, a_{9,4}=z^{\perp}, a_{14,2}=x^{\perp}, a_{14,4}=p^{\perp}
$$

or

$$
a_{9,2}=x^{\perp}, a_{9,4}=p^{\perp}, a_{14,2}=x, a_{14,4}=z^{\perp} .
$$

If the first alternative holds in both $M_{0}$ and $M_{3}$, then $A$ is not maximal since we can replace the entries $a_{6,1}$ and $a_{14,1}$ with a new vector variable and its perpendicular. Thus the first alternative cannot hold in both $M_{0}$ and $M_{3}$. Similarly, the second alternative cannot hold in both $M_{0}$ and $M_{3}$. If different alternatives hold in $M_{0}$ and $M_{3}$ then one can verify that $A$ belongs to the switching class 14. For instance, assume that (18) and (21) hold. Note that $a_{2,2}=a_{1,2}=x$ and $a_{2,4}=a_{1,4}=z^{\perp}$. By permuting the rows 3,4,5 of $N_{0}$, we may assume

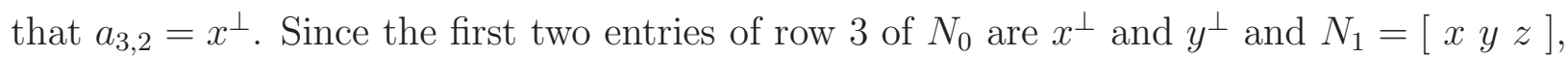
we infer that $a_{3,4}=z^{\perp}$ and $a_{4,4}=a_{5,4}=z$. Similarly, we may assume that $a_{11,2}=x$ and obtain that $a_{11,4}=p^{\perp}$ and $a_{12,4}=a_{13,4}=p$. Since $A$ is maximal, the variables $a_{4,2}, a_{12,2}, x$ must be independent. One can now verify that $A$ is equivalent to the second matrix in (17).

Finally, suppose that $u:=a_{8,3}$ and $y$ are independent. Since we have already handled the cases $a_{8,3} \in\left\{y, y^{\perp}\right\}$, we may assume that $v:=a_{8,4}$ and $z$ are independent. It follows that $x$ must have multiplicity 3 in $M_{0}$ and $M_{3}$. We may assume that the second coulmn

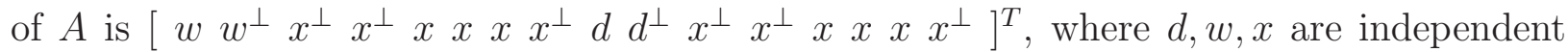
variables. Since $A[1,5,6,7 ; 3,4] \in \mathcal{O}(2), y$ or $z$ must have multiplicity 2 in this submatrix, and multiplicity 3 in $M_{0}$ since $M_{0}$ is irreducible. We may assume that $y$ has multiplicity 3 in $M_{0}$ and $u$ multiplicity 1 . As $a_{1,3}=a_{2,3}, a_{1,4}=a_{2,4}$ and $A[2,3,4,8 ; 3,4] \in \mathcal{O}(2)$, we may assume that $a_{4,3}=u^{\perp}$. Since row 8 of $A$ is orthogonal to the first three rows, we deduce that $a_{1,4}=a_{2,4}=a_{3,4}=v^{\perp}$. It follows that $a_{4,4}=v$, and we may assume that $a_{5,4}=z^{\perp}$ and $a_{6,4}=v$. Since the row 7 of $A$ is orthogonal to the first two rows, we 
deduce that $a_{1,3}=a_{2,3}=y^{\perp}$. Hence, $a_{3,3}=a_{5,3}=y$. As $A[9,13,14,15 ; 3,4] \in \mathcal{O}(2)$, $y$ or $z$ must have multiplicity 2 in this submatrix, and so multiplicity 3 in $M_{3}$. As also $A[10,13,14,15 ; 3,4] \in \mathcal{O}(2)$, we deduce that $a_{9,3}=a_{10,3}$ and $a_{9,4}=a_{10,4}$. If $y$ has multiplicity 2 in $A[9,13,14,15 ; 3,4]$, then $a_{9,3}=a_{10,3}=y$ and $a_{13,3}=a_{14,3}=y^{\perp}$. We may assume that $a_{11,3}=u^{\perp}$ and $a_{12,3}=y^{\perp}$. Thus $\pi_{3}=6,2>\pi_{1}$ and we have a contradiction. We conclude that $z$ must have multiplicity 2 in $A[9,13,14,15 ; 3,4]$. Then we must have $a_{9,4}=a_{10,4}=z^{\perp}$, and we may assume that $a_{14,4}=a_{13,4}^{\perp}=z$ and that $a_{11,4}=z, a_{12,4}=v^{\perp}$. Since rows 14 and 15 are orthogonal, we have $a_{14,3}=y^{\perp}$. Since rows 11 and 16 are orthogonal, we have $a_{11,3}=u^{\perp}$. Since rows 8 and 9 are orthogonal, we have $a_{9,3}=a_{10,3}=u^{\perp}$. Consequently, $a_{12,3}=a_{13,3}=u$. Thus $\pi_{3}=\pi_{4}=4^{2}$ and $A$ is in switching class 15 .

Thus we have shown that there are exactly 15 switching classes in $\mathcal{O}(4)$ and we have obtained the list of their representatives as given in the theorem. To complete the proof, one has to apply all possible switching operations to these 15 representatives and select the nonequivalent matrices among them. In each case there are just a few such operations. The representatives of the switching classes 4,11,12,13,14,15 admit no nontrivial switching operations. For the other switching classes we list their equivalence classes in the appendix. We omit the details.

\section{APPLICATIONS}

In this section we explain the mathematical and physical meaning and application of our results.

\section{A. Construction of OPBs in higher dimensions}

We use the 4-qubit OPBs to construct reducible 5-qubit OPBs as follows. If $\left|\alpha_{1}\right\rangle, \ldots\left|\alpha_{16}\right\rangle$ and $\left|\beta_{1}\right\rangle, \ldots\left|\beta_{16}\right\rangle$ are two 4 -qubit OPBs, then $\left|0, \alpha_{1}\right\rangle, \ldots\left|0, \alpha_{16}\right\rangle$ and $\left|1, \beta_{1}\right\rangle, \ldots\left|1, \beta_{16}\right\rangle$ are two reducible 5-qubit OPBs. Since we have classified all 4-qubit OPBs in Theorem 19, this construction covers all reducible 5-qubit OPBs. By using the same idea, we can construct all reducible $(n+1)$-qubit OPBs provided that all $n$-qubit OPBs are known.

We can construct OPBs by using the tensor product of two OPBs. Let $\mathcal{H}^{\prime}=\mathcal{H}_{1}^{\prime} \otimes$ $\cdots \otimes \mathcal{H}_{n}^{\prime}$ be another $n$-partite Hilbert space with $\operatorname{Dim} \mathcal{H}_{j}^{\prime}=d_{j}^{\prime}$ and $D^{\prime}=d_{1}^{\prime} \cdots d_{n}^{\prime}$. Let 
$\mathcal{A}^{\prime}=\left\{\left|a_{j, 1}^{\prime}, \ldots, a_{j, n}^{\prime}\right\rangle, \quad j=1, \ldots, D^{\prime}\right\} \in \mathcal{H}^{\prime}$ be an OPB. Then

$$
\mathcal{A} \otimes \mathcal{A}^{\prime}:=\left\{\left|a_{i, 1}, a_{j, 1}^{\prime}\right\rangle \otimes \cdots \otimes\left|a_{i, n}, a_{j, n}^{\prime}\right\rangle, \quad i=1, \cdots, D, \quad j=1, \cdots, D^{\prime}\right\}
$$

is an OPB of the $n$-partite Hilbert space $\otimes_{i=1}^{n}\left(\mathcal{H}_{i} \otimes \mathcal{H}_{i}^{\prime}\right)$. We have

Lemma 20 One of $\mathcal{A}$ and $\mathcal{A}^{\prime}$ is reducible if and only if $\mathcal{A} \otimes \mathcal{A}^{\prime}$ is reducible.

Proof. The "only if" part is trivial. Let us prove the "if" part. Suppose $\mathcal{A} \otimes \mathcal{A}^{\prime}$ is reducible. Without any loss of generality, we may assume that there exists a nontrivial partition $(P, Q)$ of the set $\{1, \ldots, D\} \times\left\{1, \ldots, D^{\prime}\right\}$ such that $\left|a_{j, 1}, a_{k, 1}^{\prime}\right\rangle \perp\left|a_{j^{\prime}, 1}, a_{k^{\prime}, 1}^{\prime}\right\rangle$ for all $(j, k) \in P$ and $\left(j^{\prime}, k^{\prime}\right) \in Q$. Let us set $P_{j}=\{k:(j, k) \in P\}$ and $Q_{j}=\{k:(j, k) \in Q\}$. If both $P_{j}$ and $Q_{j}$ are nonempty for some $j$, then $\mathcal{A}^{\prime}$ is reducible. Otherwise, $\mathcal{A}$ is reducible.

\section{B. Weak equivalence and controlled unitary operations}

We have introduced the weak equivalence for maximal matrices in $\mathcal{O}(n)$ in Sec. IV]. In this subsection we explain, from the viewpoint of practical implementation, why we chose the weak equivalence as the classification criterion. For example the two matrices in (9) are weakly equivalent. Here each pair, say $\left\{a, a^{\perp}\right\}$, represents a qubit o. n. basis. So the two matrices in (9) represent two families of OPBs. The second of these matrices is obtained from the first by interchanging the two columns of the lower right $4 \times 2$ submatrix. So we can convert one family to the other by the controlled unitary operation $U=|a\rangle\langle a| \otimes I_{4}+$ $\left|a^{\perp}\right\rangle\left\langle a^{\perp}\right| \otimes S_{2}$, where $S_{2}$ is the SWAP gate on two-qubit state. That is, if $\left|\alpha_{j}\right\rangle$ is a product state in the first matrix of (9) , then $U\left|\alpha_{j}\right\rangle$ is a product state in the second matrix of (9). In general, the definition of weak equivalence implies that two weakly equivalent states are convertible by a series of controlled unitaries consisting of an identity and a SWAP gate on certain qubits. The controlled unitaries can be physically implemented with a high probability and accuracy. They have been extensively investigated in recent years [12 14]. In this sense, one may experimentally implement the conversion of different OPBs using controlled unitaries. This is beneficial to quantum error correction and state preparation.

We give the formal definition as follows. A bipartite unitary gate $U$ is a controlled unitary gate if $U$ is equivalent to $\sum_{j=1}^{d_{1}}|j\rangle\langle j| \otimes U_{j}$ or $\sum_{j=1}^{d_{2}} V_{j} \otimes|j\rangle\langle j|$ via local unitaries. We say that $U$ is a controlled unitary from $A$ or $B$ side, respectively. Furthermore, $U$ is controlled in the computational basis from $A$ side if $U=\sum_{j=1}^{d_{1}}|j\rangle\langle j| \otimes U_{j}$. 


\section{DISCUSSION}

The orthogonal product bases (OPBs) in $\mathcal{H}=\mathcal{H}_{1} \otimes \mathcal{H}_{2} \otimes \cdots \otimes \mathcal{H}_{n}$ are easy to describe in the bipartite case $(n=2)$ when, say, $d_{1}=2$. (Recall that $d_{i}=\operatorname{Dim} \mathcal{H}_{i}$ and $D=\prod d_{i}$.) For this see Proposition 1 and Corollary 2. However, in general, the bipartite case remains open.

In general, the construction and the classification of OPBs up to local unitary operations reduces to the case of so called irreducible OPBs (see section II). The irreducible OPBs have been described and classified in [9] in the case of two qutrits $\left(n=2, d_{1}=d_{2}=3\right)$ and the case of three qubits $\left(n=3, d_{1}=d_{2}=d_{3}=2\right)$.

The multiqubit case $\left(d_{1}=d_{2}=\cdots=d_{n}=2\right)$ is much easier to solve than the other cases (apart from those mentioned above). Indeed, we have shown (see Sec. III) that, in the multiqubit case, the construction of OPBs reduces to a purely combinatorial problem. In the case of four qubits, we were able to solve this combinatorial problem. Our main result is that there are 33 explicit multiparameter families of OPBs of four qubits such that any $\mathrm{OPB}$ is equivalent to a member of one of these families.

We have discussed this combinatorial problem with Vijay Ganesh. In his opinion, our combinatorial problem for $n=5,6$ could be solved by using computers. It is an interesting question to discuss the computational complexity of finding the complete characterization of the OPBs of an $n$-qubit system.

Our approach to the problem of construction and classification of OPBs of $n$-qubit system is based on the classification of maximal matrices in $\mathcal{O}(n)$. As mentioned in section III, to a given OPB $\mathcal{A}:=\left\{\left|a_{s}\right\rangle=\left|a_{s, 1}, \ldots, a_{s, n}\right\rangle: s=1, \ldots, D\right\}$ we can associate a matrix say $A \in \mathcal{O}(n)$ simply by setting $A[s, j]$ to be a vector variable subject to the following conditions:

(i) $A[s, j]=A[t, j]$ if and only if $\left|a_{s, j}\right\rangle=\left|a_{t, j}\right\rangle$;

(ii) $A[s, j]=A[t, j]^{\perp}$ if and only if $\left|a_{s, j}\right\rangle=\left|a_{t, j}\right\rangle^{\perp}$;

(iii) a vector variable cannot occur in two different columns of the matrix $A$.

It is immediate from this definition that $\mathcal{A} \in \mathcal{F}_{A}$. The matrix $A$ does not have to be maximal and is not unique as we can choose the names of vector variables in many ways. However, for two equivalent OPBs their associated matrices in $\mathcal{O}(n)$ will be always equivalent. Thus we obtain a map from the set of equivalence classes of OPBs to the set of equivalence classes of matrices in $\mathcal{O}(n)$. 
If $A$ is not maximal then there exists $B \in \mathcal{O}(n)$ such that $A<B$. In that case we have $\mathcal{F}_{A} \subset \mathcal{F}_{B}$. Hence the relation $\mathcal{A} \in \mathcal{F}_{M}$, with $M \in \mathcal{O}(n)$, does not determine the equivalence class $[M]$ uniquely. This can be corrected by introducing strict families. For $M \in \mathcal{O}(n)$ we define the strict family $\mathcal{F}_{M}^{\#}$ by setting

$$
\mathcal{F}_{M}^{\#}:=\mathcal{F}_{M} \backslash \cup_{N: N<M} \mathcal{F}_{N}
$$

Then, going back to our OPB $\mathcal{A}$ and its associated matrix $A \in \mathcal{O}(n)$, we have $\mathcal{A} \in \mathcal{F}_{A}^{\#}$. Moreover, the relation $\mathcal{A} \in \mathcal{F}_{M}^{\#}$ implies that $\mathcal{F}_{M}^{\#}=\mathcal{F}_{A}^{\#}$, i.e., $\mathcal{A}$ belongs to a unique strict family, namely $\mathcal{F}_{A}^{\#}$.

\section{ACKNOWLEDGEMENTS}

We thank the two anonymous referees for their helpful comments and suggestions. Thanks to them, the paper was greatly improved. We also thank Vijay Ganesh for the discussion. LC was supported by Beijing Natural Science Foundation (4173076), the NNSF of China (Grant No. 11501024), and the Fundamental Research Funds for the Central Universities (Grant Nos. 29816133 and 74026601). The second author was supported in part by the National Sciences and Engineering Research Council (NSERC) of Canada Discovery Grant 5285.

\section{APPENDIX}

There are 15 switching classes in $\mathcal{O}(4)$. Their representatives are listed in (13)-(17). On the other hand there are 33 equivalence classes of maximal matrices in $\mathcal{O}(4)$. Each of the six switching classes $4,11,12,13,14,15$ contains a single equivalence class of maximal matrices. For each of the remaining nine switching classes, we list below the representatives $A$ of the equivalence classes of maximal matrices contained in them. We also record the number $\nu_{A}$ of independent variables that occur in the matrix $A$. This number is constant over each switching class.

Switching class 1: $\nu_{A}=15$. This switching class is the disjoint union of six equivalence 
classes.

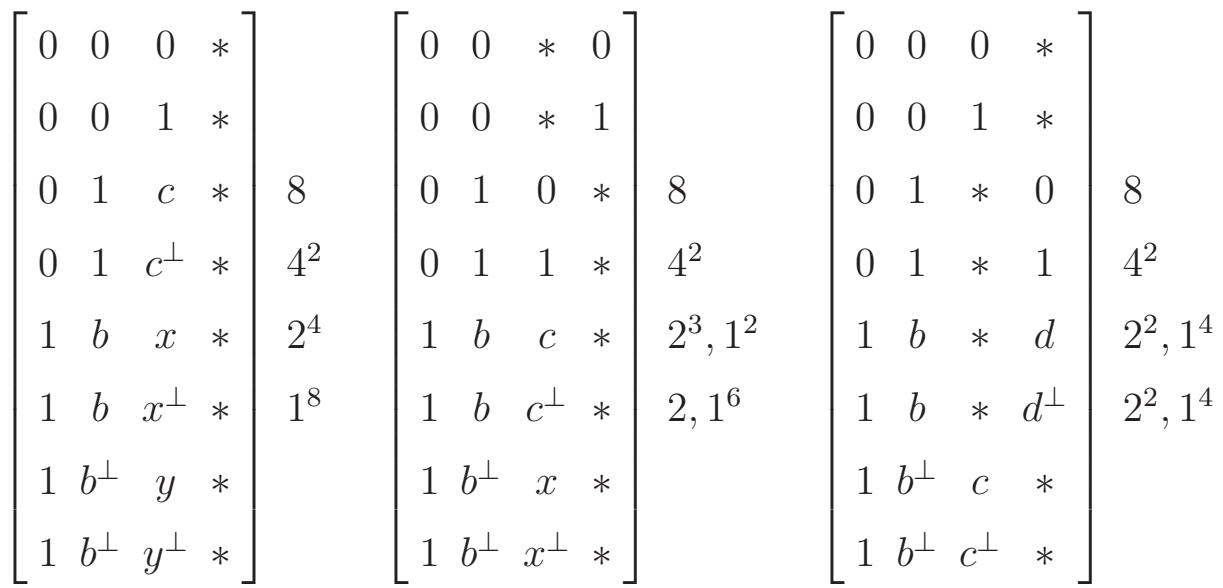

$$
\begin{aligned}
& \left.\left.\left[\begin{array}{llll}
0 & 0 & 0 & * \\
0 & 0 & 1 & * \\
0 & 1 & c & * \\
0 & 1 & c^{\perp} & * \\
1 & b & * & 0 \\
1 & b & * & 1 \\
1 & b^{\perp} & * & d \\
1 & b^{\perp} & * & d^{\perp}
\end{array}\right] 2^{2}, 1^{4}, 1^{4}\left[\begin{array}{cccc}
0 & 0 & * & 0 \\
0 & 0 & * & 1 \\
0 & 1 & c & * \\
0 & 1 & c^{\perp} & * \\
1 & b & 0 & * \\
1 & b^{\perp} & 0 & * \\
1 & x & 1 & * \\
1 & x^{\perp} & 1 & *
\end{array}\right] \begin{array}{l}
4,2^{2} \\
4,2,1^{2} \\
2,1^{6}
\end{array}\right] \begin{array}{llll}
0 & 0 & * & 0 \\
0 & 0 & * & 1 \\
0 & 1 & * & d \\
0 & 1 & * & d^{\perp} \\
1 & b & 0 & * \\
1 & b^{\perp} & 0 & * \\
1 & x & 1 & * \\
1 & x^{\perp} & 1 & *
\end{array}\right] \begin{array}{l}
2^{2}, 1^{4} \\
4,1^{4}
\end{array}
\end{aligned}
$$

Switching class 2: $\nu_{A}=14$. This switching class is the disjoint union of two equivalence classes

$$
\left.\left.\left[\begin{array}{cccc}
0 & 0 & c & * \\
0 & 0 & c^{\perp} & * \\
0 & 1 & x & * \\
0 & 1 & x^{\perp} & * \\
1 & b & 0 & 0 \\
1 & * & 1 & 0 \\
1 & b & * & 1 \\
1 & b^{\perp} & 0 & * \\
1 & b^{\perp} & 1 & 1
\end{array}\right] \begin{array}{l}
3,3,1 \\
3,2^{2}, 1 \\
0
\end{array}\right] \begin{array}{cccc}
0 & 0 & * & d \\
0 & 0 & * & d^{\perp} \\
0 & 1 & c & * \\
0 & 1 & c^{\perp} & * \\
1 & * & 1 & 0 \\
1 & b & 0 & 0 \\
1 & b & * & 1 \\
1 & b^{\perp} & 0 & * \\
1 & b^{\perp} & 1 & 1
\end{array}\right] \begin{aligned}
& \\
& 8,3,1 \\
& 3,2,1^{3} \\
& 3
\end{aligned}
$$

Switching class 3: $\nu_{A}=14$. This switching class is the disjoint union of four equivalence 
classes.

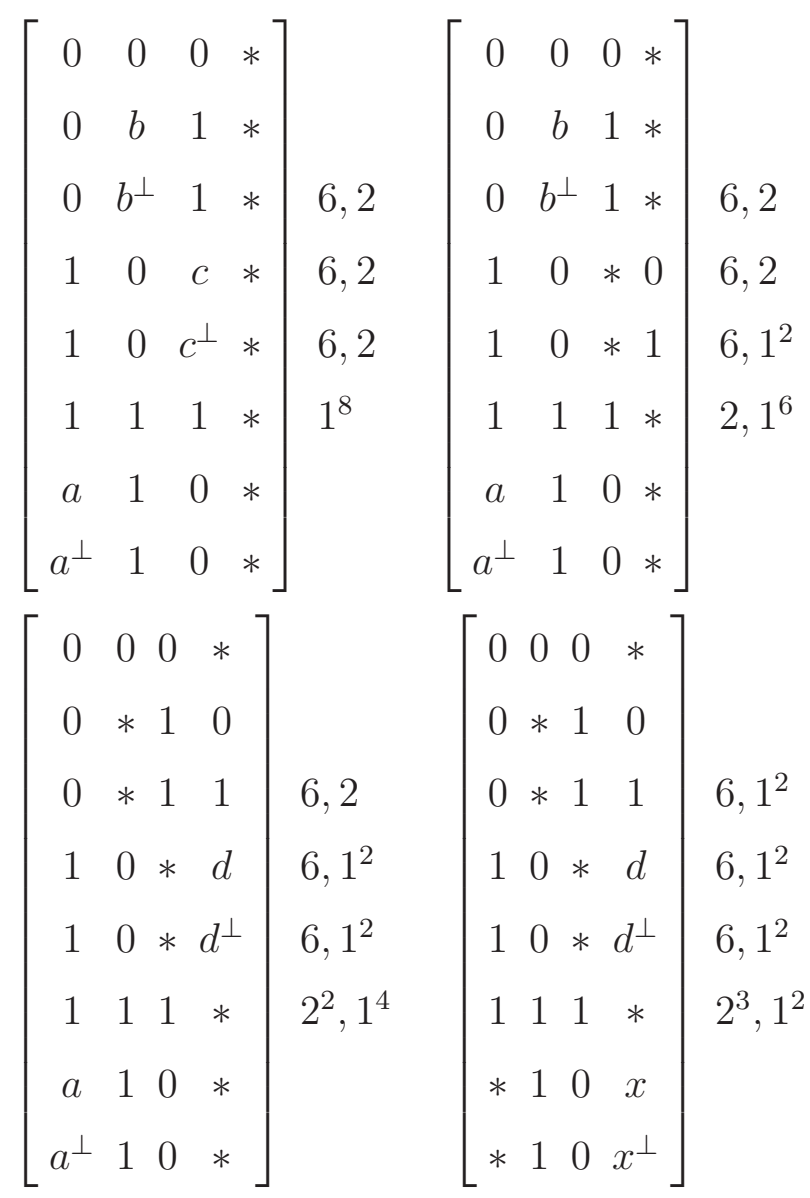

Switching class 5: $\nu_{A}=13$. This switching class is the disjoint union of four equivalence 
classes.

$$
\begin{aligned}
& {\left[\begin{array}{cccc}
0 & 0 & 0 & * \\
0 & 1 & 0 & 1 \\
0 & b & 1 & * \\
0 & b^{\perp} & 1 & * \\
1 & 1 & 1 & 0 \\
1 & 1 & * & 1 \\
1 & 0 & c & * \\
1 & 0 & c^{\perp} & * \\
* & 1 & 0 & 0
\end{array}\right] \begin{array}{l}
7,1 \\
5,2,1 \\
3,1^{5}
\end{array}\left[\begin{array}{cccc}
0 & 0 & 0 & * \\
0 & 1 & 0 & 1 \\
0 & b & 1 & * \\
0 & b^{\perp} & 1 & * \\
1 & 1 & 1 & 0 \\
1 & 1 & * & 1 \\
1 & 0 & * & d \\
1 & 0 & * & d^{\perp} \\
* & 1 & 0 & 0
\end{array}\right] \begin{array}{l} 
\\
7,1 \\
6,1^{3} \\
3,2,1^{3}
\end{array}} \\
& {\left[\begin{array}{cccc}
0 & 0 & 0 & * \\
0 & 1 & 0 & 1 \\
0 & * & 1 & d \\
0 & * & 1 & d^{\perp} \\
1 & 1 & 1 & 0 \\
1 & 1 & * & 1 \\
1 & 0 & c & * \\
1 & 0 & c^{\perp} & * \\
* & 1 & 0 & 0
\end{array}\right] \begin{array}{l}
3,2,1^{3} \\
5,2,1 \\
7,1^{2}
\end{array}\left[\begin{array}{cccc}
0 & 0 & 0 & * \\
0 & 1 & 0 & 1 \\
0 & * & 1 & d \\
0 & * & 1 & d^{\perp} \\
1 & 1 & 1 & 0 \\
1 & 1 & * & 1 \\
1 & 0 & * & x \\
1 & 0 & * & x^{\perp} \\
* & 1 & 0 & 0
\end{array}\right] \begin{array}{l} 
\\
7,1 \\
6,1^{2} \\
3,2^{2}, 1
\end{array}}
\end{aligned}
$$

Switching class 6: $\nu_{A}=13$. This switching class is the disjoint union of three equivalence classes.

$$
\left[\begin{array}{cccc}
0 & 0 & 0 & 0 \\
0 & * & 0 & 1 \\
0 & b & 1 & * \\
0 & b^{\perp} & 1 & * \\
1 & 1 & 1 & 0 \\
1 & 1 & * & 1 \\
1 & 0 & c & * \\
1 & 0 & c^{\perp} & * \\
* & 1 & 0 & 0
\end{array}\right] \begin{aligned}
& 5,1 \\
& 5,2,1 \\
& 5,1^{4}
\end{aligned}\left[\begin{array}{cccc}
0 & 0 & 0 & 0 \\
0 & 0 & * & 1 \\
0 & 1 & * & d \\
0 & 1 & * & d^{\perp} \\
1 & 1 & 1 & 0 \\
1 & * & 1 & 1 \\
1 & b & 0 & * \\
1 & b^{\perp} & 0 & * \\
* & 0 & 1 & 0
\end{array}\right] \begin{aligned}
& 7,1 \\
& 7,2,1 \\
& 5,1^{3} \\
& 4,2,1^{2}
\end{aligned}\left[\begin{array}{cccc}
0 & 0 & 0 & 0 \\
0 & * & 0 & 1 \\
0 & * & 1 & d \\
0 & * & 1 & d^{\perp} \\
1 & 1 & 1 & 0 \\
1 & 1 & * & 1 \\
1 & 0 & * & x \\
1 & 0 & * & x^{\perp} \\
* & 1 & 0 & 0
\end{array}\right] \begin{aligned}
& 5,1^{3} \\
& 5,1^{3} \\
& 4,2^{2}
\end{aligned}
$$

Switching class 7: $\nu_{A}=12$. This switching class is the disjoint union of two equivalence 
classes.

$\left.\left[\begin{array}{llll}0 & 0 & 0 & 0 \\ 0 & 0 & 1 & * \\ 0 & 1 & c & * \\ 0 & 1 & c^{\perp} & * \\ 1 & 1 & 1 & 0 \\ 1 & 1 & 0 & * \\ 1 & 0 & * & 0 \\ 1 & * & 1 & 1 \\ * & 0 & 0 & 1\end{array}\right] \begin{array}{l}7,1 \\ 4,1 \\ 0,1\end{array} \quad\left[\begin{array}{llll}0 & 0 & 0 & 0 \\ 0 & 0 & 1 & * \\ 0 & 1 & * & d \\ 0 & 1 & * & d^{\perp} \\ 1 & 1 & 1 & 0 \\ 1 & 1 & 0 & * \\ 1 & 0 & * & 0 \\ 1 & * & 1 & 1 \\ * & 0 & 0 & 1\end{array}\right] \begin{array}{l} \\ 7,1 \\ 7,1^{3} \\ \end{array}\right]$

Switching class 8: $\nu_{A}=12$. This switching class is the disjoint union of two equivalence classes.

$\left.\left.\left[\begin{array}{llll}0 & 0 & 0 & 0 \\ 0 & 1 & 0 & d \\ 0 & * & 1 & d \\ 0 & 1 & * & d^{\perp} \\ 0 & 0 & 1 & d^{\perp} \\ 1 & 1 & c & * \\ 1 & 1 & c^{\perp} & * \\ 1 & 0 & 1 & 1 \\ 1 & 0 & * & 0 \\ * & 0 & 0 & 1\end{array}\right] \begin{array}{l}7,2,1 \\ 3^{2}, 1^{2} \\ 0\end{array}\right] \begin{array}{cccc}0 & 0 & 0 & 0 \\ 0 & 1 & 0 & d \\ 0 & * & 1 & d \\ 0 & 1 & * & d^{\perp} \\ 0 & 0 & 1 & d^{\perp} \\ 1 & 1 & * & x \\ 1 & 1 & * & x^{\perp} \\ 1 & 0 & 1 & 1 \\ 1 & 0 & * & 0 \\ * & 0 & 0 & 1\end{array}\right] \begin{gathered} \\ 7,1 \\ 7,1 \\ 3^{2}, 2 \\ \end{gathered}$

Switching class 9: $\nu_{A}=12$. This switching class is the disjoint union of two equivalence 
classes.

$$
\left[\begin{array}{cccc}
0 & 0 & 0 & 0 \\
0 & b & 0 & 1 \\
0 & b & 1 & * \\
0 & b^{\perp} & 1 & 0 \\
0 & b^{\perp} & * & 1 \\
1 & 1 & 0 & 1 \\
1 & 1 & 1 & * \\
1 & 0 & d & * \\
1 & 0 & d^{\perp} & * \\
* & 1 & 0 & 0
\end{array}\right] \begin{aligned}
& 5,1 \\
& 4,1^{4} \\
& 0
\end{aligned} \quad\left[\begin{array}{cccc}
0 & 0 & 0 & 0 \\
0 & b & 0 & 1 \\
0 & b & 1 & * \\
0 & b^{\perp} & 1 & 0 \\
0 & b^{\perp} & * & 1 \\
1 & 1 & 0 & 1 \\
1 & 1 & 1 & * \\
1 & 0 & * & d \\
1 & 0 & * & d^{\perp} \\
* & 1 & 0 & 0
\end{array}\right] \begin{aligned}
& 5,1 \\
& 5,1^{3} \\
& 4,1^{2} \\
&
\end{aligned}
$$

Switching class 10: $\nu_{A}=12$. This switching class is the disjoint union of two equivalence classes.

$\left.\left.\left[\begin{array}{llll}0 & 0 & 0 & 0 \\ 0 & 1 & 1 & 1 \\ 0 & * & 1 & 0 \\ 0 & 0 & * & 1 \\ 1 & 1 & 1 & d \\ 1 & 0 & 0 & d^{\perp} \\ 1 & * & 1 & d^{\perp} \\ 1 & 0 & * & d \\ a & 1 & 0 & * \\ a^{\perp} & 1 & 0 & *\end{array}\right] \begin{array}{l}3^{2}, 1^{2} \\ 0,1^{2} \\ 0\end{array}\right] \begin{array}{llll}0 & 0 & 0 & 0 \\ 0 & 1 & 1 & 1 \\ 0 & * & 1 & 0 \\ 0 & 0 & * & 1 \\ 1 & 1 & 1 & d \\ 1 & 0 & 0 & d^{\perp} \\ 1 & * & 1 & d^{\perp} \\ 1 & 0 & * & d \\ * & 1 & 0 & x \\ * & 1 & 0 & x^{\perp}\end{array}\right] \begin{aligned} & 6,1^{2} \\ & 3^{2}, 2 \\ & 6,1^{2} \\ & \end{aligned}$

[1] E. Chitambar, D. Leung, L. Mancinska, M. Ozols, A. Winter, Everything You Always Wanted to Know About LOCC (But Were Afraid to Ask), Commun. Math. Phys. 328, 303 (2014).

[2] C. H. Bennett, G. Brassard, C. Crépeau, R. Jozsa, A. Peres, and W. K. Wootters, Phys. Rev. Lett. 70, 1895 (1993).

[3] D. P. DiVincenzo, P. W. Shor, J. A. Smolin, B. M. Terhal, and A. V. Thapliyal, Phys. Rev. A 61, 062312 (2000).

[4] W. Dür, J. I. Cirac, M. Lewenstein, and D. Bruss, Phys. Rev. A 61, 062313 (2000). 
[5] D. DiVincenzo, T. Mor, P. Shor, J. A. Smolin, and B. M. Terhal, Commun. Math. Phys. 238, $379(2003)$.

[6] C. H. Bennett, D. P. DiVincenzo, C. A. Fuchs, T. Mor, E. Rains, P. W. Shor, J. A. Smolin, and W. K. Wootters, Quantum nonlocality without entanglement, Phys. Rev. A, 59, 1070 (1999).

[7] L. Chen and D. Ž. Đoković, Boundary of the set of separable states, Proc. R. Soc. A 471: 20150102.

[8] R. F. Werner, Quantum states with einstein-podolsky-rosen correlations admitting a hiddenvariable model, Phys. Rev. A 40, 4277 (1989).

[9] Y. Feng and Y. Shi, Characterizing locally indistinguishable orthogonal product states, IEEE Trans. Inform. Theory 55 (2009).

[10] C. H. Bennett, D. P. DiVincenzo, T. Mor, P. W. Shor, J. A. Smolin, and B. M. Terhal, Unextendible product bases and bound entanglement, Phys. Rev. Lett. 82, 5385 (1999).

[11] S. B. Bravyi, "Unextendible Product Bases and Locally Unconvertible Bound Entangled States", Quant. Inf. Process. 3, 309 (2004).

[12] L. Chen and L. Yu, Phys. Rev. A89, 062326 (2014).

[13] L. Chen and L. Yu, On the Schmidt-rank-three bipartite and multipartite unitary operator, Annals of Physics, 351, 682 (2014).

[14] L. Chen and L. Yu, Entanglement cost and entangling power of bipartite unitary operators of Schmidt rank three (2015), 1507.05260.

[15] D. McNulty, B. Pammer, and S. Weigert, J. Math. Phys.57, 032202 (2016). 